\title{
Les fondateurs de la population de La Patrie (Cantons-de-l'Est) : Franco-américains, Québécois et Européens aux recensements canadiens de 1881 et de 1891 The biological founders of the population of La Patrie in the Eastern Townships : Franco-Americans, Québécois and Europeans in the 1881 and 1891 Canadian Censuses
}

\section{Francine M. Mayer et Mireille Boisvert}

Volume 39, numéro 2, automne 2010

URI : https://id.erudit.org/iderudit/1003589ar

DOI : https://doi.org/10.7202/1003589ar

Aller au sommaire du numéro

Éditeur(s)

Association des démographes du Québec

ISSN

0380-1721 (imprimé)

1705-1495 (numérique)

Découvrir la revue

Citer cet article

Mayer, F. M. \& Boisvert, M. (2010). Les fondateurs de la population de La Patrie (Cantons-de-l'Est) : Franco-américains, Québécois et Européens aux

recensements canadiens de 1881 et de 1891. Cahiers québécois de démographie, 39(2), 307-356. https://doi.org/10.7202/1003589ar

\section{Résumé de l'article}

Ce travail constitue le premier volet d'un large programme de recherche interdisciplinaire sur la contribution des immigrants franco-américains au patrimoine génétique de la population des Cantons de l'Est, et plus particulièrement à celui de la population de La Patrie fondée en 1875 dans le cadre du mouvement de rapatriement des Canadiens français émigrés aux États-Unis. Afin de bien comprendre les interactions entre les facteurs biodémographiques et socioculturels qui ont façonné les pools géniques de celle-ci, nous avons fait une première analyse transversale et comparative des recensements nominatifs canadiens de 1881 et 1891 . Ces derniers suivent de près la création de la colonie de rapatriement et leur analyse permet d'identifier les premiers fondateurs biologiques de la population et d'obtenir une première estimation du nombre de familles fondatrices franco-américaines qui représentent plus du tiers des ménages à chaque recensement. Les structures démographiques montrent une population jeune et un rapport de masculinité élevé pour le groupe d'âge des 10-39 ans qui suggère que les familles immigrantes sont arrivées avec plus de fils que de filles en âge de travailler au défrichement et à l'ensemencement des lots concédés. Les alternances dans la déclaration du lieu de naissance des membres du ménage révèlent une mobilité importante d'un nombre de familles immigrantes, qui font de fréquents allers-retours transfrontaliers. Les professions sont plus diversifiées au recensement de 1891, avec toujours une prédominance de l'agriculture alors que le travail lié à la coupe de bois offre une soupape de sûreté importante pour les familles.
Tous droits réservés @ Association des démographes du Québec, 2010
Ce document est protégé par la loi sur le droit d'auteur. L'utilisation des services d’Érudit (y compris la reproduction) est assujettie à sa politique d'utilisation que vous pouvez consulter en ligne. 


\title{
Les fondateurs de la population de La Patrie (Cantons-de-l'Est): Franco-américains, Québécois et Européens aux recensements canadiens de 1881 et de 1891
}

\author{
FRANCINE M. MAYER ET MIREILLE BOISVERT ${ }^{*}$
}

\begin{abstract}
Ce travail constitue le premier volet d'un large programme de recherche interdisciplinaire sur la contribution des immigrants franco-américains au patrimoine génétique de la population des Cantons de l'Est, et plus particulièrement à celui de la population de La Patrie fondée en 1875 dans le cadre du mouvement de rapatriement des Canadiens français émigrés aux ÉtatsUnis. Afin de bien comprendre les interactions entre les facteurs biodémographiques et socioculturels qui ont façonné les pools géniques de celle-ci, nous avons fait une première analyse transversale et comparative des recensements nominatifs canadiens de 1881 et 1891. Ces derniers suivent de près la création de la colonie de rapatriement et leur analyse permet d'identifier les premiers fondateurs biologiques de la population et d'obtenir une première estimation du nombre de familles fondatrices franco-américaines qui représentent plus du tiers des ménages à chaque recensement. Les structures démographiques montrent une population jeune et un rapport de masculinité élevé pour le groupe d'âge des 10-39 ans qui suggère que les familles immigrantes sont arrivées avec plus de fils que de filles en âge de travailler au défrichement et à l'ensemencement des lots concédés. Les alternances dans la déclaration du lieu de naissance des membres du ménage révèlent une mobilité importante d'un nombre de familles immigrantes, qui font de fréquents allers-retours transfrontaliers. Les professions sont plus diversifiées au recensement de 1891, avec toujours une prédominance de l'agriculture alors que le travail lié à la coupe de bois offre une soupape de sûreté importante pour les familles.
\end{abstract}

English abstract, p. 356

* Département des sciences biologiques, Université du Québec à Montréal. Cette recherche a été financée par une subvention du Conseil de recherche en sciences humaines du Canada (2005-2009), octroyée à Francine M. Mayer dans le cadre du programme de recherche intitulé "Contributions des immigrants franco-américains au patrimoine génétique de la population des Cantons-de-l'Est (Québec) : les fondateurs de La Patrie aux XIX ${ }^{\mathrm{e}}$ et $\mathrm{XX}^{\mathrm{e}}$ siècles ». Les auteurs remercient les évaluateurs externes de la première version du manuscrit, leurs critiques et conseils ayant, à leur avis, aidé à améliorer la version finale. 


\section{INTRODUCTION}

\section{Problématique}

Dans un contexte de colonisation, les premiers immigrants venus s'établir sont habituellement ceux dont la contribution au patrimoine génétique de la population ${ }^{1}$ sera la plus importante, de sorte qu'un certain nombre d'individus de la population à un moment donné de son histoire vont partager un ou plusieurs de ces ancêtres. Ceci s'explique par le fait que la migration est souvent de type familial et que, en sus de leur propre famille, ces premiers immigrants sont parfois accompagnés d'un frère ou d'un beaufrère marié, d'un fils avec femme et enfants ou encore d'un gendre avec sa propre famille. Les colons qui s'établissent dans la communauté après cette vague initiale de peuplement épousent en bonne partie les enfants des premiers immigrants et contribuent, par leur propre fécondité, à augmenter, en même temps que leur propre représentation génétique, celle de ces derniers. Tous ces immigrants, quelle que soit la date de leur arrivée dans la population et quelle que soit l'importance de leur contribution au patrimoine génétique de la population, sont considérés comme étant ses fondateurs biologiques ${ }^{2}$. La contribution de ces fondateurs est tributaire de leur fécondité et de celle de leurs descendants, ainsi que des choix matrimoniaux de ces derniers. En effet, les unions préférentielles entre apparentés ou entre lignées endogames vont multiplier, au cours des générations, les liens qui relient le fondateur à ses descendants et vont augmenter la représentation relative de ses gènes par rapport à celle des autres fondateurs. La fécondité du fondateur et celle de ses descendants seront aussi modulées par les ponctions faites sur sa descendance par les décès des enfants avant l'âge de la reproduction, par les célibats définitifs, par la stérilité de certains couples et par le départ de la communauté non seulement de jeunes adultes en âge de procréer, mais aussi de familles entières. La taille de la famille "utile» est un concept qui correspond au nombre d'enfants du couple qui seront incorporés dans un couple à la

1. Dans une perspective évolutive, la population correspond à une communauté d'individus appartenant à une espèce à reproduction sexuée et au sein de laquelle ces individus se reproduisent (Dobzhansky, 1970 cité dans Wapples et Caggiotti, 2006). L'isolat théorique correspond à l'unité minimum de population dans laquelle on a quelque droit de penser que la panmixie prévaut ou existe (Dahlberg, 1929 cité dans Gomila, 1971).

2. Sont considérés ici comme fondateurs tous les individus immigrants dans la communauté et qui se trouvent, en fin d'information, dans les chaînes d'ascendance de tous les individus de la population pour toutes les périodes étudiées (Jacquard, 1972). 
génération suivante (Jacquard et Nadot, 1968 ; Jacquard, 1977). C'est à partir de ces descendants « utiles » que sera mesurée la contribution génétique du fondateur, à l'aide du calcul de probabilité d'origine des gènes ${ }^{3}$. La taille de la famille « utile » est aussi la résultante des interactions entre les composantes biodémographiques de chaque cellule biologique qui constitue le noyau familial et les différents facteurs sociaux, culturels et économiques qui interviendront dans son histoire et dans celle de la communautét.

\section{Objectifs du programme de recherche}

Cette problématique alimente l'ensemble de notre démarche de recherche, dont l'objectif global est l'étude des contributions des immigrants francoaméricains au patrimoine génétique de la population des Cantons-de-l'Est aux $\operatorname{xix}^{\mathrm{e}}$ et $\mathrm{xx}^{\mathrm{e}}$ siècles, et plus particulièrement celles des fondateurs de La Patrie. Elle vise non seulement à mesurer la participation de ces derniers aux pools géniques successifs de la population, en tenant compte des contributions des diverses régions du Québec à ceux-ci, mais aussi à décortiquer le rôle des dynamiques socioculturelles, économiques et environnementales dans la structuration du patrimoine génétique. Ce programme de recherche ambitieux a plusieurs volets, dont cet article constitue la première étape. Les sources utilisées, de même que les approches méthodologiques adoptées, ont dicté le découpage des étapes de cette recherche. Chacune de ces dernières constitue donc un tout indépendant, ayant des objectifs spécifiques et dont les résultats offrent une base sur laquelle peuvent s'appuyer, du moins en partie, les démarches ultérieures.

3. La probabilité d'origine des gènes est la " probabilité qu'un gène pris au hasard chez un fondateur se retrouve chez les individus de la population étudiée " (Jacquard, 1972). Elle peut être calculée à l'aide de la formule (Heyer, 1999) :

$$
\sum_{i=1}^{p} \sum_{j=1}^{c}\left(\frac{1}{2}\right)^{g_{i j}}
$$

où $p$ est le nombre de descendants du fondateur; $c$, le nombre de chemins généalogiques entre le fondateur et un individu et $g_{i j}$, le nombre de générations séparant le fondateur $i$ pour un chemin $j$. Comme il s'agit d'un calcul de probabilité, le total des contributions ne peut dépasser l'unité.

4. Il y a plusieurs définitions de la communauté, dont celle de Laura Thompson (1967) qui, selon Gomila (1971), voyait l'isolat concret dans « la population locale en transaction avec l'écosystème naturel dont elle forme une partie perçue comme un événement changeant dans l'espace-temps ». 


\section{Cadre de référence du programme de recherche}

Notre démarche globale s'inscrit dans le prolongement des études réalisées sur la communauté de La Patrie par P. Nadon (1970) et F. M. Mayer (1977), certains questionnements soulevés alors trouvant aujourd'hui réponse grâce, entre autres, à l'accès à de nouvelles sources. Il faut rappeler que ces premiers travaux ont été réalisés dans le cadre d'une large étude comparative de petites communautés rurales isolées ${ }^{5}$ dont la principale caractéristique était d'être des paroisses de colonisation de fondation relativement récente, ce qui permettait de remonter aux fondateurs et de saisir les modalités d'apparition de la consanguinité. Il s'agissait de mener pour chaque communauté une étude ethnologique susceptible de fournir le schéma expérimental définitif permettant de bien identifier les barrières délimitant les échanges génétiques pour chaque communauté. À cette étude s'ajoutait une perspective diachronique fournie par la constitution d'un Registre de la population, registre réalisé à l'aide du jumelage des actes d'états civils contenus dans les registres paroissiaux de manière à pouvoir mener une analyse sociodémographique exhaustive de la population et suivre l'évolution de sa structure génétique (Gomila et Guyon, 1969).

Dans son analyse ethnologique de la communauté de La Patrie, Nadon caractérise cette dernière par une relation économique étroite avec les États-Unis, et ce, dès le début de la colonie. Il met en évidence l'existence de mouvements transfrontaliers pour une partie de sa population, par l'entremise ou non de la parenté, et l'analyse des structures démographiques à l'aide du Registre de la population (Mayer, 1977) confirme l'importance de la migration. Compte tenu des sources utilisées, il était impossible d'identifier les allers-retours et de les attribuer directement à des individus ou à des familles particulières. Le Registre a cependant permis de démontrer que les migrations en tant qu'événements démographiques ont été plus importantes que le mouvement naturel de la population. L'étude de la nuptialité a pris en compte le phénomène migratoire, l'analyse de la

5. Ce programme de recherche, financé par le Conseil des Arts du Canada, a été entrepris en 1966 par les professeurs du département d'anthropologie de l'Université de Montréal, J. Benoist, G. Dubreuil et J. Gomila. Cinq des sept communautés étudiées étaient de peuplement québécois dont quatre au Québec et une en Alberta. Cette dernière et celle des Cantons-de-l'Est ont été fondées par des Canadiens français précédemment émigrés aux États-Unis. Les deux autres, situées à l'Île-du-PrinceÉdouard et au Nouveau-Brunswick, sont d'origine acadienne. Plusieurs mémoires et thèses ont été réalisés dans le cadre de ce programme entre 1968 et 1977. 
composition des unions ne pouvant se limiter à une seule observation des unions endogames et exogames. L'étude du comportement des couples face à l'émigration a en effet démontré que les couples formés d'immigrants quittaient la communauté plus que les autres couples, en y laissant rarement un enfant déjà marié. La comparaison des relevés démographiques aux effectifs des recensements canadiens depuis 1871 a montré une sous-estimation de l'immigration pour un peu plus que les trois premières décennies et une importante sous-estimation de l'émigration pour les périodes suivantes, et ce, jusqu'à la fin de la période d'observation. L'importance du mouvement migratoire rendait donc obsolète l'étude des structures génétiques par le biais de l'étude de la consanguinité. Par contre, le calcul de la probabilité d'origine des gènes (Jacquard, 1972) s'est révélé beaucoup plus adapté, puisqu'il a permis d'évaluer la contribution relative des fondateurs de la population, quelle que soit la date de leur arrivée et quelle que soit la pérennité de leur représentation à travers leur descendance. Les 833 couples fondateurs (1 404 individus) identifiés par l'analyse génétique ont produit en moyenne 1,35 fratrie par couple, confirmant la nature ouverte de la population, plutôt qu'une population de type isolat. Le succès reproducteur se mesure alors par la persévérance de la représentation dans les pools géniques successifs plutôt que par le nombre de descendants. En effet, parmi ces couples, seulement $25 \%$ étaient encore représentés à travers leur descendance au recensement ethnographique de 1968 et, de ce nombre, $11 \%$ étaient d'immigration récente et donc toujours vivants en 1968 ! Une émigration aussi importante ramène à peu la contribution des fondateurs, même si deux couples d'immigrants arrivés au début de la colonie sont à la tête de deux lignées endogames avec des représentations probabilistes relativement importantes. Quelle était la position de ces familles immigrantes dans la stratification sociale qui, selon Nadon, remonterait au début de la colonie et oppose agriculteurs et bûcherons? Quel rôle ont-elles joué dans le passage d'une économie d'échange à une économie de marché? Ce passage a restreint, selon Nadon, l'influence de la famille et de la parenté dans l'organisation sociale de la communauté, sans toutefois éliminer l'entraide et la collaboration entre parents, mises alors au service de la réussite économique. Quelles sont la position des immigrants venus des États-Unis et celle de leurs descendants dans cette dynamique socio-économique? Où se situent les autres immigrants? Bien que l'enquête ethnographique ait permis de préciser dans plusieurs cas le statut de franco-américain des premiers immigrants, l'origine des fondateurs n'a pas pu être prise en compte dans l'analyse de leurs contributions génétiques, puisque cette information n'était pas systématique, n'étant pas 
toujours connue des membres de la communauté et étant rarement enregistrée dans les actes d'état civil. L'enquête de terrain a bien mis en évidence l'importance des liens de parenté et de la famille dans l'organisation sociale de la communauté, mais elle n'a pas pu examiner ces deux variables dans le contexte de la migration de départ des États américains ou des paroisses du Québec, et encore moins en mesurer l'ampleur. Le Registre de la population n'est pas plus bavard, puisque dans le cadre d'une immigration familiale les couples arrivent déjà mariés. Les filiations consignées dans l'acte de mariage figurent dans les livres des paroisses d'origine, ce qui rend impossible l'identification des liens de parenté entre les immigrants, quoique ces liens aient été partiellement précisés dans certains écrits historiques (Annuaire du Séminaire, 1897-1898).

Les interrogations soulevées par ces deux études ont alimenté notre problématique de recherche sur la représentation différentielle des fondateurs de la population, problématique très pertinente dans le contexte actuel de recherches théoriques et appliquées qui consacrent beaucoup d'efforts et de ressources à identifier et caractériser les pools géniques régionaux du Québec (CARTaGÈNE, 2009). Nous connaissons déjà bien les modalités de peuplement des régions du Québec (voir par exemple Charbonneau, 1991) et des chercheurs ont analysé les rôles joués par l'économie agro-forestière, par la situation socio-économique des familles, par la parenté et par les réseaux d'alliance dans la stratification sociale, le comportement migratoire et l'établissement des familles dans les nouveaux terroirs (voir entre autres Séguin, 1977; Bouchard, 1978; Maisonneuve, 1985; Mathieu et collab., 1987; Dessureault, 2001). En revanche, le rôle des immigrants franco-américains dans la colonisation des Cantons-de-l'Est et leur impact sur le pool génique de la région est mal connu. Compte tenu de ses spécificités, la population de La Patrie constitue un objet pertinent pour approfondir notre démarche, d'autant plus que de nombreuses sources maintenant disponibles viennent enrichir le corpus de données déjà en place, rendant aujourd'hui réalisable ce qui tenait du rêve scientifique il y peu de temps encore. Les premiers colons établis dans la colonie de La Patrie sont-ils effectivement ceux dont la contribution aux pools géniques successifs est la plus importante? Quels sont les facteurs explicatifs du phénomène? Les immigrants franco-américains sont-ils surreprésentés dans le patrimoine génétique de la population, sont-ils rapidement retournés dans l'est des États-Unis ou ont-ils émigré vers l'Ouest américain ou les provinces canadiennes de l'ouest? L'origine franco-américaine de la communauté est-elle encore une réalité biologique, ou n'est-elle plus qu'une réalité historique? Jusqu'à quel point et surtout de quelle façon la 
parenté a-t-elle été un moteur d'immigration au départ des villes américaines et une porte de sortie définitive ou temporaire de la colonie dans les périodes économiquement éprouvantes? Le maintien des liens avec la famille américaine a-t-il perduré au-delà des premières générations, et se reflète-t-il dans le choix du conjoint, dans la distribution spatiale des établissements et dans les voisinages? Le know how des Franco-américains revenus au Québec (Ramirez et Lamarre, 1985) les aurait-il avantagés socialement et économiquement et, si oui, de quelle manière dans un contexte dominé par l'agriculture et les activités liées à l'industrie du bois?

\section{Approche méthodologique et sources}

Comme nous l'avons précisé en introduction, chaque étape de la recherche fait appel à des sources et des méthodes différentes ou combinées. Il serait lourd d'élaborer ici chacune des approches anticipées pour la réalisation de l'ensemble des objectifs du programme. Elles accompagneront chaque projet spécifique et leur pertinence y sera alors discutée et démontrée. Rappelons simplement ici qu'afin de bien comprendre les mécanismes modulant l'histoire évolutive de la population, il faut à la fois se donner un outil longitudinal d'analyse sous la forme de généalogies ascendantes et descendantes, afin de suivre la transmission génétique des fondateurs, et identifier les liens de parenté entre les fondateurs de la population. Le Registre de la population fournit déjà les descendances à partir des fondateurs, de même que les ascendances jusqu'à ces derniers. Nous devons donc reconstituer à cette étape les ascendances des fondateurs en ayant recours aux enregistrements civils religieux des autres paroisses du Québec et des États-Unis, de même qu'aux enregistrements civils des États américains. La consultation des recensements canadiens et américains est également essentielle pour cette reconstitution. Les listes nominatives sont d'une richesse documentaire exceptionnelle pour analyser les dynamiques sociales qui ont prévalu à chaque période. Elles offrent une ethnographie de la communauté pour les périodes qui ne sont pas accessibles à l'enquête directe (Benoist, 1989). Le jumelage des données transversales entre elles et avec les données longitudinales fournies par le Registre de population permet d'inscrire dans l'espace l'histoire des familles et des généalogies (Lavoie et collab., 1988) et de documenter la transmission différentielle des gènes en tenant compte des dynamiques liées aux changements.

Étant donné nos questions de départ et les outils à notre disposition, l'analyse transversale et comparative des recensements doit être réalisée en premier. Elle permet de connaître la structure démographique et sociopro- 
fessionnelle de la population ainsi que ses effectifs par âge et par sexe, et elle fournit une estimation plus précise de l'imperfection des données longitudinales à mesurer la migration. La déclaration des lieux de naissance donne une première estimation du nombre de ménages francoaméricains, européens et québécois, de même que celui des familles impliquées dans les allers-retours transfrontaliers. L'analyse de la composition des ménages brosse un tableau de l'organisation sociale en fonction de l'origine des familles et des métiers exercés. Avant de présenter les résultats de ces premières analyses, il convient de rappeler le contexte historique de fondation de la communauté.

\section{CONTEXTE DU RAPATRIEMENT}

L'adoption, le 23 février 1875, par le gouvernement du Québec, de l'Acte de rapatriement fut le premier effort officiel fait pour convaincre les émigrés francophones du Québec partis aux États-Unis, plus particulièrement ceux des villes industrielles de la Nouvelle-Angleterre, de revenir au Québec et de s'établir comme colons sur les terres de la Couronne. La colonisation était considérée par les élites, durant les dernières décennies du $\mathrm{xIx}^{\mathrm{e}}$ siècle, comme la seule façon de réintégrer les expatriés. Selon Linteau (2000), les mesures officielles de rapatriement n'auraient cependant pas remporté le succès escompté et bon nombre de Franco-américains ayant tenté l'aventure du retour au Québec vers les régions de colonisation seraient repartis assez rapidement aux États-Unis. De plus, ces mesures n'auraient touché qu'une infime partie de ceux qui sont revenus des États-Unis, les sociétés de colonisation ayant réussi de leur côté à convaincre un certain nombre de familles de retourner au Québec. Il faut ajouter les rapatriements spontanés, qui pourraient expliquer de nombreux retours vers les paroisses du Québec d'où les familles étaient parties initialement. Riches de leur expérience industrielle acquise aux États-Unis, ces familles ont pu aussi s'établir dans les villes du Québec et alimenter le mouvement d'exode rural vers les centres urbains.

Selon Ramirez (1991), le choix d'une stratégie migratoire relève le plus souvent de la famille, s'appuie sur les réseaux de parenté et de voisinage et dépend des perspectives et des aspirations individuelles et familiales, de la conjoncture économique, de l'âge des personnes, de leur état civil, de la taille et de la composition des familles et enfin de la présence de connaissances ou de parents sur le lieu d'arrivée. Ramirez et Lamarre (1985) considèrent que l'émigration devrait être étudiée en prenant en compte les 
relations socio-économiques impliquant autant le lieu de départ que le lieu de destination, d'autant plus que le phénomène migratoire a souvent donné lieu à des mouvements de retour susceptible de canaliser des capitaux et du know how et ayant sans doute influé sur l'évolution de la société d'origine. Toujours dans cette perspective, plusieurs auteurs précisent qu'il ne faut pas négliger non plus les phénomènes d'allers-retours, comme en témoignent les naissances en alternance d'enfants nés d'un côté ou l'autre de la frontière, phénomène fréquemment observé (Anctil, 1980 ; Ramirez, 1991; Rodrigue, 1997).

\section{LA PATRIE, COLONIE DE RAPATRIEMENT}

Dans son article portant sur La Patrie en 1875-188o, John I. Little (1977) rappelle que les efforts de rapatriement, dans le cadre de l'Acte de rapatriement, se sont concentrés dans trois cantons du district de Saint-François : Chesham, Emberton et surtout Ditton (La Patrie), ces cantons étant la seule colonie de rapatriement dans le cadre du programme gouvernemental. Ces derniers étant encore peu occupés, relativement accessibles par train et situés près de la frontière américaine. Des sociétés de colonisation financées par des fonds publics avaient déjà commencé le défrichement de lots et la construction de routes sur ces terres. La majorité des colons de Ditton seraient en fait des ruraux venus d'autres régions du Québec. Quelques centaines seulement seraient des expatriés, la plupart repartant aux ÉtatsUnis en 1879 dès le rétablissement de la prospérité économique là-bas. Les colons proviendraient principalement du reste des Cantons-de-l'Est, puis du Lac-Saint-Jean-Saguenay, ce qui signifierait, selon Little, que déjà à cette date ces deux zones de colonisation étaient saturées. La récession ayant touché les villes canadiennes dès 1875, 47 familles de colons de Ditton seraient venues des villes de Montréal, Québec, Trois-Rivières, Sherbrooke ou Coaticook. Le recensement de la population de Ditton, fait le 16 avril 1875, dénombre 308 colons formant 54 familles. Ils sont originaires d'Europe (124 individus), du Québec (110 individus) et des États-Unis (74 individus) et sont arrivés entre 1863 et 1874, donc dans le cadre des activités des sociétés de colonisation. Le 31 octobre 1876 , les colons en provenance des États américains représentent $51 \%$ de la population (498 sur 969, voir Annuaire du Séminaire 1896-1899 et note 86 dans Little, 1977). Les conditions de colonisation sont difficiles et plusieurs terres ne se prêtent pas à l'agriculture, forçant les colons à chercher des emplois en foresterie, ce qui les pousse à négliger le travail d'ensemencement de leurs champs. En mai 
1877 , le statut de la colonie comme centre de rapatriement est officiellement terminé, la crise dans les villes n'encourageant guère le gouvernement à favoriser le retour des Franco-américains au Québec. En 1889, le département des terres de la Couronne est forcé d'admettre que les prêts faits aux rapatriés pour défricher leurs lots ne seront jamais remboursés et, en 1898, il annule la dette. Mais, selon Little, ce montant n'aurait pas été investi en vain. La population des trois cantons n'a pas décliné une fois le projet terminé : en fait, elle a légèrement augmenté, passant de 1927 personnes en 1877 à 2112 en 1880 . Les récoltes sont notables et les trois cantons se prévalent de trois églises et deux curés, cinq écoles, cinq magasins, un hôtel, trois municipalités très bien organisées et 65 milles de routes, dont une menant à la station de chemin de fer de Scotstown. Même si beaucoup de lotissements n'ont pas été le résultat direct du programme de rapatriement, tous ont été stimulés par les fonds versés dans la région par l'entremise de ce projet. Nadon (1970) reconnaît que l'essor démographique très rapide de La Patrie est la résultante de la vague de colonisation qui déferle sur les Cantons-de-l'Est, du mouvement de rapatriement lancé aux États-Unis et de l'émergence, en même temps que celle de la communauté, d'un grand nombre de scieries, l'industrie du bois assurant le maintien d'une population relativement importante, dont une bonne proportion était auparavant flottante.

\section{CHOIX DES RECENSEMENTS DE 1891 ET DE 1881 ET VARIABLES PERTINENTES À L'ANALYSE}

Marc St-Hilaire (1996) écrit qu'il faut environ 25 ans pour qu'une population perde son statut de colonie et devienne une communauté. Le choix du recensement de 1891 comme point de départ des analyses semble approprié puisque les premiers colons arrivent à La Patrie en 1863 (Annuaire du séminaire, 1897-1898 et 1898-1899) : en 1891, la période pionnière serait donc terminée. Le recensement de 1881 permet, quant à lui, non seulement d'analyser les fondateurs encore en période de colonisation, mais également d'enrichir notre compréhension des phénomènes observés, en ajoutant une perspective comparative. Le recensement de 1891 nous servira aussi de charnière pour une comparaison ultérieure avec le recensement de La Patrie de 1901.

Bien que le recensement de 1891, comme celui de 1881 avant lui, ne donne pas le patronyme de l'épouse, il indique, contrairement à ce dernier, les liens entre le chef de ménage et les autres membres du ménage, ce qui est un avantage pour les analyses généalogiques. De plus, les lieux de nais- 
sance des parents de chacun des individus recensés sont donnés. Les recensements de 1881 et de 1891 partagent sensiblement les mêmes rubriques, mais celui de 1891 offre des informations supplémentaires (tableau 1).

TABLEAU 1 Rubriques du recensement de 1891

\begin{tabular}{|c|c|}
\hline 1 Bâtiments et chantiers & 14 Lieu de naissance de la mère \\
\hline 2 Maisons en voie de construction & 15 Religion \\
\hline 3 Maisons inhabitées & 16 Profession, occupation ou métier \\
\hline 4 Maisons habitées & 17 Patrons \\
\hline 5 Familles & 18 Employés \\
\hline 6 Noms & $\begin{array}{l}19 \text { Sans emploi durant la semaine } \\
\text { précédant le recensement }\end{array}$ \\
\hline 7 Sexe & $\begin{array}{l}20 \text { Nb de pers. employées par les patrons } \\
\text { durant l'année }\end{array}$ \\
\hline 8 Âge & 21 Sachant lire \\
\hline 9 Marié ou en veuvage & 22 Sachant écrire \\
\hline 10 Lien de parenté avec le chef de famille & 23 Sourds-muets \\
\hline 11 Pays ou province de naissance & 24 Aveugles \\
\hline 12 Canadiens français & 25 Atteint d'aliénation mentale \\
\hline 13 Lieu de naissance du père & \\
\hline
\end{tabular}

En gras: Rubrique non présente en 1881

Le recensement de 1891 a été dépouillé et informatisé à partie d'une copie imprimée du microfilm, puis vérifié en ligne (ancestry.com), compte tenu du fait que la qualité du microfilm laissait à désirer, particulièrement les bas de pages impaires, pratiquement illisibles pour les 3 ou 4 dernières lignes. Quant au recensement de 1881, déjà indexé par individu et rendu partiellement accessible en ligne (colonnes 6 à 15), par les mormons (Family Search/Genealogical Society of Utah), il a été validé à partir d'une copie du microfilm. Les deux recensements de la population de La Patrie ont eu lieu du 4 au 26 avril 1881 et 16 au 22 avril 1891. Le recenseur, Pierre Louis Napoléon Prévost, était le même aux deux recensements; résident de La Patrie, il en était le maire en 1881. Il connaissait donc bien la population recensée.

Le nombre de maisons inhabitées (tableau 2) apparaît très important aux deux recensements, bien qu'il ne surprenne pas vraiment : en effet, Little (1977) mentionne que, dès 1877 , un gardien avait été engagé pour prévenir le démantèlement des maisons abandonnées. 
TABLEAU 2 Nombre de maisons inhabitées et habitées aux recensements de 1881 et de 1891

\begin{tabular}{|l|c|c|}
\hline & Recensement de 1881 & Recensement de $\mathbf{1 8 9 1}$ \\
\hline Maisons inhabitées/maisons construites & $62 / 202$ & $41 / 189$ \\
\hline Maisons habitées/nombre de ménages & $140 / 154$ & $148 / 153$ \\
\hline Nombre d'individus/nombre de ménages & $898 / 154$ & $828 / 153$ \\
\hline
\end{tabular}

En 1881, les 62 maisons inhabitées ${ }^{6}$ ne peuvent pas être attribuées à des secteurs particuliers. Cependant, plusieurs d'entre elles sont en rang et contiguës ( 3 séries de 6 maisons, 2 de 3 et 9 de 2), situation qu'on ne rencontre pas en 1891. En 1891, les 41 maisons inhabitées sont réparties en alternance avec les maisons habitées et elles se concentrent dans deux secteurs. Il est impossible de déterminer, aussi tôt dans les analyses, si la diminution en 1891 du nombre de maisons inhabitées est due au retour des familles ou à l'établissement de nouvelles familles. Ces maisons devaient être enregistrées à mesure que l'énumérateur les rencontrait, ce qui donne une indication des voisinages mais ne renseigne pas sur leurs propriétaires. Ce nombre important de maisons inhabitées ( $31 \%$ en $1881,22 \%$ en 1891 ) est toutefois révélateur d'un phénomène de grande ampleur qui pourrait être en lien avec les mouvements d'allers-retours aux États-Unis.

Les maisons habitées ${ }^{7}$ en 1891 sont toutes en bois et comportent 1 à 3 étages et de 2 à 12 chambres. Cette dernière information n'a pas été enregistrée en 1881, mais il est légitime de supposer que plusieurs de ces maisons étaient déjà construites lors du recensement de 1881. Les 148 maisons habitées en 1891 accueillent 153 ménages, 9 d'entre eux étant répartis dans 4 maisons; en 1881, les 140 maisons habitées abritaient 154 ménages, dont 27 sont répartis dans 13 maisons. Le partage d'une maison entre plusieurs

6. Les instructions (Département de l'agriculture, 1881 ; 1891) sont les mêmes pour les deux recensements. « Doivent être enregistrées [...] les maisons inhabitées à mesure que l'énumérateur les rencontre : avec ceci de particulier que la ligne sur laquelle le chiffre est inscrit importe peu attendu qu'il n'y a aucun rapport entre cet enregistrement et les noms et autres renseignements des autres colonnes du tableau [...]. Lorsque ces maisons [...] se rencontrent en rang et contiguës, l'enregistrement doit se faire en inscrivant le chiffre indicatif du nombre collectif. »

7. Les instructions sont claires à ce sujet : «Ici doivent être enregistrées les maisons habitées dans l'ordre des visites, consécutivement de 1 à la fin de la série pour chaque division d'énumérateur. Une maison peut contenir plusieurs familles, mais il faut toujours dans ce cas n'enregistrer qu'une maison : pour constituer deux ou plusieurs maisons d'une construction contiguë, il faut que chaque partie, ainsi prise pour une maison séparée ait une porte de dehors séparée. » 
ménages est moins fréquent avec les années. L'analyse transversale ne permet pas de présumer si ces arrangements sont basés sur la parenté ou sur l'origine géographique.

Les 153 ménages recensés en 1891 comptent 828 individus, alors que les 154 ménages de 1881 en comptaient 898 . L'un des ménages de 1891 fournit peu d'information et ne sera pas inclus dans les analyses ${ }^{8}$. Ces dernières porteront donc sur les 154 ménages de 1881 et sur 152 ménages en 1891 . Nous retiendrons le terme ménage même là où le recensement utilise le terme de famille, les deux étant équivalents dans le contexte du recensement canadien. Le manuel contenant les instructions aux officiers du deuxième recensement du Canada (1881) et à ceux du recensement de 1891 précise que : «Une famille, dans le sens attaché à ce mot pour les fins du recensement, peut n'être composée que d'une seule personne vivant seule et, d'autre part, d'un nombre quelconque de personnes vivant ensemble sous le même toit et nourries à la même cuisine [...]. La famille pour le recensement se constitue par le domicile, et le chef de famille s'entend du père, de la mère, du maître ou de la maîtresse de la maison, quel qu'il soit. » Le recenseur « [...] doit enregistrer le nombre de familles visitées, dans l'ordre des visites, en numérotant 1, 2, 3, etc. sans interruption jusqu'à la fin.»

En 1891, le recenseur a relevé 110 patronymes différents auprès des 152 ménages recensés. Bien qu'un certain nombre de chefs de ménage puissent être apparentés, la diversité des patronymes est un indicateur des origines variées des familles et des individus. Les 154 ménages recensés en 1881 partageaient 134 patronymes différents, dont 62 sont toujours présents en 1891, mais plus de la moitié (72/134) ne s'y trouvent plus; par ailleurs, $43 \%(47 / 110)$ des noms de famille relevés en 1891 se sont ajoutés entre les deux énumérations. Ces informations confirment un mouvement de population important entre les deux recensements, ainsi que la stabilité probable de certaines familles, sans présumer pour autant de celle de chacun de ses membres. La consultation des sources d'état civil permettra ultérieurement de nuancer ces observations, puisque le nom de jeune fille des épouses et veuves sera connu.

8. Le chef, avec nom et prénom inscrits, est âgé de 31 ans, déclaré marié et catholique et il habite une maison de bois; on ne dispose d'aucune autre information sur lui. Suite à cet enregistrement, une ligne vide a été laissée pour l'épouse. Cette inscription du couple n'est ni complétée ni raturée. Il s'agit fort probablement de deux individus absents. 
La différence entre le nombre de maisons inhabitées et les arrangements résidentiels observés aux deux recensements, tout comme la variété des patronymes et leur fluctuation, suggèrent des mouvements importants de population. Il s'agit maintenant de voir ce que la structure de la population révèle dans ce contexte.

\section{STRUCTURE DE LA POPULATION}

\section{Âge et sexe des individus recensés}

Le découpage par tranches de 10 ans permet d'isoler les enfants qui ne sont pas encore en âge de travailler à temps plein sur l'exploitation familiale (tableau 3). Le regroupement des deux premières classes d'âge (0-19 ans) indique que les deux populations (1891 et 1881) sont jeunes et représentent respectivement $56 \%(461 / 826)$ et $57 \%(512 / 898)$ de la population recensée, alors que les 20-59 ans en composent plus du tiers. Les 60 ans et plus sont plus nombreux en 1891, ce qui s'explique, du moins en partie, par le fait que la période de colonisation active est terminée et qu'une partie des colons immigrants recensés en 1881 ont vieilli à La Patrie. En période de colonisation, les familles dont le chef est plus âgé ne seront pas tentées de s'établir sur le front pionnier, puisque les fils en âge de travailler auront déjà majoritairement quitté la famille. Le groupe des 10-29 ans, qui comprend surtout des enfants et des célibataires non mariés, est plus fortement représenté en 1881 (362/898: $40 \%)$ qu'en 1891 (295/826:36\%), ce qui appuierait aussi l'argument du passage de la colonie au statut de communauté.

TABLEAU 3 Structure par âge et sexe de la population recensée en 1891 et en 1881 (\%)

\begin{tabular}{|l|c|c|c|c|}
\hline \multirow{2}{*}{ Groupes d'âge } & \multicolumn{2}{|c|}{$\mathbf{1 8 9 1}$} & \multicolumn{2}{c|}{$\mathbf{1 8 1}$} \\
\cline { 2 - 5 } & Hommes & Femmes & Hommes & Femmes \\
\hline 0-9 ans & 35,3 & 36,2 & 33,6 & 34,1 \\
\hline $10-19$ ans & 20,3 & 19,8 & 22,4 & 24,0 \\
\hline $20-29$ ans & 14,5 & 16,8 & 17,5 & 16,8 \\
\hline $30-39$ ans & 12,1 & 10,8 & 10,6 & 11,3 \\
\hline $40-49$ ans & 5,8 & 6,8 & 6,7 & 6,4 \\
\hline $50-59$ ans & 5,1 & 5,0 & 6,4 & 4,6 \\
\hline $60-69$ ans & 4,9 & 3,0 & 1,9 & 2,1 \\
\hline 70 ans et plus & 1,9 & 1,5 & 0,9 & 0,7 \\
\hline Total & $\mathbf{1 0 0}$ & $\mathbf{1 0 0}$ & $\mathbf{1 0 0}$ & $\mathbf{1 0 0}$ \\
\hline Effectifs & $\mathbf{4 2 8}$ & $\mathbf{3 9 8}$ & $\mathbf{4 6 4}$ & $\mathbf{4 3 4}$ \\
\hline
\end{tabular}




\section{Âge et lieu de naissance des individus recensés}

La distribution des individus par groupes d'âge et selon leur pays ou province de naissance renseigne indirectement sur la période d'arrivée des individus dans le canton (tableau 4). Les périodes de séjours aux ÉtatsUnis peuvent être, du moins en partie, déduites de la variable du lieu de naissance en lien avec l'âge déclaré.

TABLEAU 4 Structure par âge de la population recensée en 1891 et en 1881 selon le lieu de naissance

\begin{tabular}{|c|c|c|c|c|c|c|c|c|}
\hline \multirow[t]{2}{*}{ Groupes d'âge } & \multicolumn{4}{|c|}{1891} & \multicolumn{4}{|c|}{1881} \\
\hline & Tous & Québec & $\begin{array}{l}\text { États- } \\
\text { Unis }\end{array}$ & Europe & Tous & Québec & $\begin{array}{l}\text { États- } \\
\text { Unis }\end{array}$ & Europe \\
\hline $0-9$ ans & 295 & 259 & 35 & 1 & 304 & 246 & 58 & o \\
\hline $10-19$ ans & 166 & 122 & 43 & $1^{\mathrm{a}}$ & 208 & $158^{\mathrm{b}}$ & 33 & 17 \\
\hline $20-29$ ans & 129 & 111 & 16 & 2 & 154 & $136^{c}$ & 11 & 7 \\
\hline 30-39 ans & 95 & 83 & 5 & $7^{\mathrm{a}}$ & 98 & 80 & 3 & 15 \\
\hline $40-49$ ans & 52 & 47 & 1 & 4 & 59 & 45 & 3 & 11 \\
\hline $50-59$ ans & 42 & 40 & o & 2 & 50 & 42 & o & 8 \\
\hline $60-69$ ans & 33 & 27 & o & 6 & 18 & 14 & o & 4 \\
\hline 70 et plus & 14 & 14 & o & o & 7 & 6 & o & 1 \\
\hline TOTAL & 826 & 703 & 100 & 23 & 898 & 727 & 108 & 63 \\
\hline
\end{tabular}

a Deux cas de naissance hors de l'Europe: une mère et sa fille nées en Algérie

b Deux individus nés en Ontario

c Un individu né en Ontario

La distribution par groupes d'âge des individus nés au Québec suit celle de l'ensemble de la population de Ditton : ils représentent respectivement $85 \%$ et $81 \%$ de la population en 1891 et 1881 . Cette information ne permet pas de déterminer un intervalle de temps au cours duquel la majorité des familles auraient pu immigrer à La Patrie.

Quant aux individus nés aux États-Unis, ils forment $12 \%$ de la population à chaque recensement, dont $78 \%$ et $84 \%$ de moins de 20 ans. Les moins de 30 ans constituent $94 \%$ des personnes nées dans les États américains durant les périodes 1852-1881 et 1862-1891. Nous ne pouvons présumer de la date d'arrivée aux États-Unis des parents de la majorité des individus qui y sont nés, mais il est clair qu'ils y ont résidé au cours des périodes correspondant aux dates d'intensification du mouvement d'émigration aux États-Unis au milieu du xix ${ }^{\mathrm{e}}$ siècle, mouvement qui atteindra un 
sommet en 1880-189o (Lavoie, 1973). Il n'est donc pas surprenant qu'il n'y ait pas d'individus âgés de 50 ans et plus qui soient nés aux États-Unis. Cela nous amène à conclure que les individus de ces groupes d'âge établis dans la colonie étaient pour la majorité des individus nés au Québec, certains d'entre eux ayant émigré aux États-Unis puis étant revenus au Québec avec une partie de leur famille née en sol américain.

Les individus nés en Europe sont au nombre de 23 en 1891 et ne constituent que $2,7 \%$ de cette population, alors qu'en 1881 ils représentaient $7,0 \%$ de la population (63 individus). Quelques années plus tôt (octobre 1876 ) ils formaient $10,2 \%$ (99/969) de la population et 40,3 \% (124/308) en avril 1875 (Annuaire du Séminaire, 1896-1899). Il faut rappeler qu'ils représentaient $100 \%$ de la population du canton avant 1870 (Nadon, 1970). Les familles de colons européens s'étant établies plus tôt dans le canton, un certain nombre de leurs enfants figurent parmi les naissances au Québec: les individus de 0-19 ans originaires d'Europe ne représentaient plus en 1891 que $8,7 \%$ de la population d'origine européenne, contre $27,0 \%$ en 1881. Il n'est pas non plus étonnant que les individus ayant déclaré être nés dans un pays d'Europe soient plus nombreux dans les trois derniers groupes d'âge aux recensements de 1891 et 1881 (34,8 \% et 20,6 \%) comparativement aux individus nés au Québec du même groupe d'âge ( $11,5 \%$ et $8,5 \%)$. La diminution radicale des effectifs suggère des départs de la communauté et l'absence de nouvelles entrées en provenance des pays européens. L'ancienneté de l'immigration de ce groupe n'explique cependant pas à elle seule la distribution des effectifs par groupes d'âge. Par exemple, les 10-19 ans en 1881 auraient dû fournir des effectifs au groupe de 20-29 ans dix ans plus tard, mais on ne compte alors que 2 individus dans ce groupe, ce qui laisse supposer des départs vers les États-Unis. La diminution importante des individus de 30-49 ans au recensement de 1891 signifie, de la même façon, un départ de la communauté des colons européens, que l'on peut rattacher au fait qu'un bon nombre de colons anglais et norvégiens, recrutés en 1869 par la société de colonisation de Compton sous le patronage de l'homme d'affaires J. H. Pope, auraient profité en 1875-1876 de la demande en terres défrichées pour vendre leur ferme et s'établir ailleurs (Little, 1977).

Les caractéristiques des individus recensés en 1881 et 1891 nous ont donné un instantané de la population. La prise en compte des unités de recensement et de leurs caractéristiques donne quant à elle un aperçu de la structure et de l'organisation sociale de cette population. 


\section{Âge et statut matrimonial des individus déclarés chefs de ménage en 1891 et 1881}

Selon G. Bouchard (1992), les couples des paroisses saturées migrent vers le front pionnier pour acquérir de plus grandes surfaces afin d'établir leurs garçons comme agriculteurs. Il y voit deux explications possibles : soit ces familles étaient proches de la phase de transmission familiale, soit le nombre de garçons était tel que le père ne pouvait pas les établir sur la terre familiale alors qu'ils étaient assez grands pour participer à la mise en valeur des terres neuves. Lorsqu'il considère la migration dans son contexte de destination, Marc St-Hilaire (1996) observe, à partir de données longitudinales, que les couples qui se dirigent vers les zones de colonisation représentent des concentrations supérieures à la moyenne chez les plus de 40 ans, alors que les départs du contexte pionnier touchent particulièrement les couples de moins de 40 ans. Le découpage transversal qu'offrent les recensements ne livre pas toute l'information sur l'histoire reproductive de la famille et sur son itinéraire migratoire, mais il offre une perspective différente dans l'interprétation des résultats (tableau 5).

\section{Groupes d'âge des chefs de ménage}

La distribution des chefs de ménage par groupes d'âge est similaire d'un recensement à l'autre, les chefs de ménage de moins de 40 ans représentant $52 \%$ des chefs tant en 1891 qu'en 1881, et les 30-39 ans formant le groupe d'âge le plus représenté (31 \% et $29 \%$ ). Les chefs de ménage de 40 ans et plus sont par ailleurs un peu moins nombreux (48\%), contrairement à ce qui est observé au Saguenay (St-Hilaire, 1996). Cependant, comme ce groupe est principalement représenté par des chefs âgés de 40 à 59 ans, plus nombreux en 1881 (42\%) qu'en 1891 (32\%), ces observations iraient dans le sens de celles de Marc St-Hilaire, à moins que cela puisse s'expliquer par un départ un peu plus marqué de familles plus jeunes dans la période intercensitaire, familles qui ne seront pas comptabilisées 10 ans plus tard dans le groupe des 40 ans et plus. Le front pionnier aurait recruté peu de chefs âgés : $6 \%$ dans le groupe des 60 ans et plus en 1881. En 1891, ils forment $16 \%$ des chefs de ménage, un certain nombre faisant probablement partie de la population recensée en 1881 . Ces chiffres vont dans le sens de l'hypothèse d'une communauté qui vient de franchir l'étape pionnière de son développement et qui a conservé une partie de ses effectifs initiaux, qui y vieillissent. 
Statut matrimonial des chefs de ménage et taille moyenne de la famille

La majorité des chefs de ménage sont des hommes mariés. Le nombre de chefs de ménage en veuvage est le même aux deux dates, mais inclut davantage de veuves en 1891, ce qui explique le nombre supérieur de femmes chefs de ménage en 1891. Les chefs célibataires sont peu nombreux ( 6 en $1891 ; 8$ en 1881) et ce sont principalement des hommes, à l'exception de 2 institutrices à chaque recensement. La taille moyenne des ménages change peu pour la tranche des 30-59 ans, la naissance d'enfants compensant pour le départ des aînés, et ce, aux deux dates considérées. Cette stabilité pourrait être aussi en partie la conséquence d'une migration constante, touchant principalement des familles qui disposent d'enfants en âge de travailler.

TABLEAU 5 Distribution des chefs de ménage par groupe d'âge, selon le sexe et le statut matrimonial aux recensements de 1891 et de 1881

\begin{tabular}{|l|c|c|c|c|c|}
\hline Groupe d'âge 1891 & Effectifs & Hommes & Femmes & Mariés & $\begin{array}{c}\text { Taille moyenne } \\
\text { du ménage }\end{array}$ \\
\hline Moins de 20 ans & 0 & 0 & 0 & 0 & 0 \\
\hline 20-29 ans & 32 & 30 & 2 & 27 & 3,69 \\
\hline $30-39$ ans & 47 & 47 & 1 & 45 & 6,38 \\
\hline $40-49$ ans & 26 & 23 & 3 & 22 & 6,92 \\
\hline 50-59 ans & 22 & 21 & 1 & 19 & 5,64 \\
\hline 60 ans et plus & 25 & 24 & 1 & 24 & 4,16 \\
\hline Total & $\mathbf{1 5 2}^{\mathbf{a}}$ & $\mathbf{1 4 4}$ & $\mathbf{8}$ & $\mathbf{1 3 7}$ & $\mathbf{5 , 4 3}$ \\
\hline
\end{tabular}

\begin{tabular}{|l|c|c|c|c|c|}
\hline Groupe d'âge 1881 & Effectifs & Hommes & Femmes & Mariés & $\begin{array}{c}\text { Taille moyenne } \\
\text { du ménage }\end{array}$ \\
\hline Moins de 20 ans & 3 & 2 & 1 & 1 & 1,33 \\
\hline $20-29$ ans & 33 & 32 & 1 & 31 & 4,12 \\
\hline $30-39$ ans & 44 & 44 & 0 & 19 & 6,10 \\
\hline 40-49 ans & 33 & 32 & 1 & 29 & 6,06 \\
\hline $50-59$ ans & 32 & 31 & 1 & 29 & 6,03 \\
\hline 60 et plus & 9 & 9 & 0 & 7 & 4,08 \\
\hline Total & $\mathbf{1 5 4}$ & $\mathbf{1 5 0}$ & $\mathbf{4}$ & $\mathbf{1 3 7}$ & $\mathbf{5 , 8 2}$ \\
\hline
\end{tabular}

a 3 veufs, 6 veuves, 4 hommes et 2 femmes célibataires

b 7 veufs, 2 veuves, 6 hommes et 2 femmes célibataires 


\section{Rapport de masculinité des célibataires en 1891 et 1881}

Nous inspirant du travail de Marc St-Hilaire (1996) sur la colonisation au Saguenay, nous avons calculé le rapport de masculinité pour tous les individus recensés dans les ménages qui ne sont pas déclarés mariés ou veufs. Contrairement à cet auteur, nous ne disposons pas de données longitudinales et nous ne pouvons faire l'hypothèse que les familles qui émigrent vers le front pionnier sont celles qui ont davantage de fils que de filles. En revanche, nous pouvons constater que les familles qui se sont établies dans le canton de Ditton à la fin du xix ${ }^{\mathrm{e}}$ siècle y ont immigré accompagnées de plus de fils que de filles, les aînées ayant pu ne pas migrer avec le reste de la famille afin de conserver un emploi ou parce qu'elles étaient déjà mariées et établies. La proximité des États américains de la Nouvelle-Angleterre renforce l'idée d'une migration partielle des familles, certains membres s'ajustant rapidement en fonction de l'évolution de la situation économique de part et d'autre de la frontière.

Le rapport de masculinité a été calculé pour les individus de 9 ans et moins et pour ceux de 10-39 ans, étant donné qu'à partir de 10 ans la majorité des enfants travaillaient déjà (tableau 6). Cette classe d'âge comprend principalement les enfants du ménage en âge d'être impliqués dans les travaux domestiques et de la ferme et les jeunes adultes travaillant sur l'exploitation familiale et dans le secteur économique de l'industrie forestière. Elle peut inclure des célibataires jeunes et moins jeunes travaillant comme engagés salariés. Quant aux enfants de 9 ans et moins, ils ne travaillent pas à plein temps pour l'exploitation familiale et le rapport de masculinité de cette classe d'âge ne devrait pas être influencé par les considérations précédentes. Cependant, le hasard de la distribution des sexes au fur et à mesure des naissances au sein des familles a pu influencer les stratégies migratoires de la famille dans une perspective d'établissement des enfants, particulièrement des fils, y compris les derniers nés au moment du départ.

Les résultats semblent confirmer cette hypothèse. Au recensement de 1891, le rapport de masculinité chez les 9 ans et moins est de 104,8 garçons pour 100 filles et il est de 120,0 si on ne tient compte que des 10-39 ans. Le rapport de masculinité en 1881, pour chacun des groupes d'âge examiné, est encore plus élevé (respectivement 105,4 et 123,0). Bien que les effectifs soient peu nombreux, il est difficile d'attribuer cette distribution des rapports de masculinité au seul hasard. 
TABLEAU 6 Rapport de masculinité pour les groupes d'âge 0-9 ans et 10-39 ans dans la population recensée en 1891 et en 1881

\begin{tabular}{|l|c|c|c|c|c|c|}
\hline \multirow{2}{*}{$\begin{array}{l}\text { Groupes } \\
\text { d'âge }\end{array}$} & \multicolumn{3}{|c|}{1891} & \multicolumn{3}{c|}{1881} \\
\cline { 2 - 7 } & $\begin{array}{c}\text { Nombre } \\
\text { de garçons }\end{array}$ & $\begin{array}{c}\text { Nombre } \\
\text { de filles }\end{array}$ & $\begin{array}{c}\text { Rapport de } \\
\text { masculinité }\end{array}$ & $\begin{array}{c}\text { Nombre } \\
\text { de garçons }\end{array}$ & $\begin{array}{c}\text { Nombre } \\
\text { de filles }\end{array}$ & $\begin{array}{c}\text { Rapport de } \\
\text { masculinitéa }\end{array}$ \\
\hline O-9 ans & 151 & 144 & $\mathbf{1 0 4 , 8}$ & 156 & 148 & $\mathbf{1 0 5 , 4}$ \\
\hline $10-39$ ans & 126 & 105 & $\mathbf{1 2 0 , 0}$ & 155 & 126 & $\mathbf{1 2 3 , 0}$ \\
\hline
\end{tabular}

a (garçons/filles) $\times 100$

La perspective longitudinale qu'offre la comparaison des rapports de masculinité entre les deux recensements suggère une population avec plus d'hommes aux âges auxquels la main-d'œuvre est la plus active. Les emplois disponibles demeurent toujours principalement masculins en 1891 : les cinq scieries de La Patrie en pleine activité en 1890 (Nadon 1970) embauchent surtout des hommes, ce qui permet de maintenir sur place les familles qui ont plusieurs fils avec elles et ce qui attire de nouvelles familles de même profil.

\section{DÉPARTAGE DES MÉNAGES QUÉBÉCOIS ET FRANCO-AMÉRICAINS}

La distribution par groupes d'âge et selon le lieu de naissance des individus recensés donne les effectifs de Franco-américains, d'Européens et de Québécois en 1891 et 1881 . C'est cependant l'analyse des ménages au sein desquels les individus sont recensés qui va permettre de mieux comprendre le pattern migratoire des familles. Dans son étude sur les migrants américains et franco-américains au Québec, Linteau (2000) reconnaît que le terme «franco-américain » est flou dans sa définition et il retient pour son étude la définition la moins restrictive : "Tous les Canadiens français et tous leurs descendants ayant séjourné où que ce soit aux États-Unis ou y étant nés et qui sont ensuite venus vivre au Québec de façon temporaire ou permanente ». Nous avons adapté cette définition et avons défini un ménage comme étant franco-américain si l'un de ses membres au moins est déclaré né aux États-Unis. Il peut s'agir du chef de ménage, de son épouse, d'un enfant ou de plusieurs enfants recensés à la suite du chef de ménage, d'un étranger recensé en dernier ou encore d'un ou plusieurs membres du ménage formant une extension, ou d'individus appartenant au noyau secondaire du ménage.

Cette définition est limitative, et ne permet pas d'inclure un ménage dont tous les membres recensés sont nés au Québec mais qui ont pu 
séjourner aux États-Unis avant de s'établir à La Patrie. Ces familles fondatrices vont pouvoir ultérieurement dans la recherche être considérées comme franco-américaines, lorsque leur provenance sera confirmée. Rappelons en effet que certains des chefs de ménage recensés en 1881 sont inscrits sur la liste d'énumération des premiers immigrants à La Patrie réalisée en avril 1876 (Annuaire du séminaire, 1897-1898). Cette liste donne le nom de la ville américaine d'où les premières familles immigrantes sont venues, ainsi que le nombre d'individus composant chaque famille et les numéros de lots attribués. Alors que le recensement de 1881 est totalement muet quant au séjour américain de ces familles, la liste confectionnée en 1876 confirme cette réalité. Prenons le cas hypothétique d'un ménage dont le chef est déclaré né aux États-Unis au recensement de 1891, et qui a immigré adolescent à La Patrie, donc recensé dans le ménage de ses parents en 1881. Devrait-il être considéré au recensement de 1891 comme étant un ménage franco-américain? Pour complexifier le jeu, ce même fils a pu travailler de l'autre côté de la frontière entre les deux recensements, et même s'y être marié avant de revenir s'établir et fonder sa famille à La Patrie. Dans ce dernier cas, le ménage qu'il forme devrait être considéré comme franco-américain. En fait, seuls les jumelages entre les données nominatives des recensements, les jumelages avec les mentions de l'état civil constituées en Registre de population, la reconstitution des ascendances des couples recensés sur quelques générations et le rapprochement des mentions avec diverses autres sources pourront confirmer le statut de ménage franco-américain et identifier les stratégies migratoires des familles et de leurs membres. Les familles originaires des vieilles paroisses et des villes du Québec pourront alors être mieux départagées de celles qui ont séjourné aux États-Unis.

Les listes nominatives demeurent une source incomparable pour la compréhension des diverses stratégies migratoires empruntées par les familles. En nous indiquant le lieu de naissance du chef et de son épouse ainsi que celui des enfants, elles permettent d'estimer minimalement le nombre de ménages immigrants selon leur provenance et de classer les ménages selon les types de trajectoires migratoires grâce à l'alternance dans les déclarations du lieu de naissance des enfants. De plus, la prise en considération des intervalles entre l'âge déclaré de chacun des membres du ménage peut aussi suggérer des allers-retours de chaque côté de la frontière. Un décès infantile enregistré dans la paroisse franco-américaine pourrait expliquer un plus long intervalle associé à un séjour relativement court de la famille aux États-Unis. Un intervalle long peut être interprété soit par le départ d'un jeune adulte par mariage ou pour des 
raisons d'emploi à l'extérieur du canton, soit par un séjour hors frontière de la famille qui laissera derrière elle un jeune adulte du ménage. Les écarts d'âge déclaré au sein du ménage peuvent aussi identifier des cas de remariage et suggérer des déplacements du ménage plus complexes qu'il n'y paraît de prime abord. L'âge des parents combiné au nombre d'enfants dans le ménage est révélateur de la phase du cycle familial sans livrer pour autant la provenance du ménage. L'examen des écarts d'âge et des intervalles entre naissances aide à quantifier les incertitudes du départage des ménages selon leur provenance et la trajectoire migratoire, mais il ne peut que suggérer des scénarii qu'il est impossible de retenir comme valides sans reconstitution des familles et jumelage de différentes sources.

\section{Classification des ménages selon le lieu de naissance de ses membres}

Théoriquement, plusieurs permutations sont possibles quant aux lieux de naissance des membres du ménage, puisqu'il peut y avoir, au rythme des migrations de la famille, une alternance dans la déclaration du lieu de naissance des enfants. De plus, trois combinaisons parentales sont possibles, sans compter les lieux de naissance déclarés des membres du ménage qui constituent une extension de la famille ou un noyau secondaire dans le cas des ménages complexes.

Afin de diminuer le nombre de combinaisons à analyser, les ménages ont été classés selon le lieu de naissance du chef de ménage et de son épouse, puis selon les différentes permutations du lieu de naissance des enfants ou de celui des membres ajoutés à la cellule nucléaire. Rappelons que le nombre d'individus dans le ménage dépend de la phase atteinte par le cycle familial lors du recensement ainsi que du départ de certains de ses membres. Rappelons également que plus la taille du ménage est grande, plus les alternances dans la déclaration du lieu de naissance sont possibles, et que les ménages avec peu d'enfants ou sans enfant sont très peu loquaces.

Les 152 ménages recensés en 1891 se répartissent comme suit : 137 (90 \%) ménages ont un chef né au Québec et 15 ménages (10 \%) un chef né soit aux États-Unis, soit en Europe (France, Norvège, Irlande, Belgique). Les 154 ménages recensés en 1881 comprennent 130 ménages dont le chef est né aux Québec ( $84 \%$ ) et 24 ménages ( $16 \%$ ) dont le chef est né aux États-Unis ou en Europe (Angleterre, France, Norvège, Écosse, Irlande, Belgique). 


\section{Ménage dont le chef est né aux États-Unis ou en Europe}

En 1891, les 15 ménages en titre semblent regroupés selon leur origine européenne ou américaine si l'on se fie aux numéros de ménage (non illustré ici) ${ }^{9}$. L'âge moyen des 10 chefs nés en Europe est de 49,3 ans, alors que celui des 5 chefs nés aux États-Unis est de 31,0 ans. Les chefs nés aux États-Unis ont, ou ont eu, un conjoint né au Québec. Les chefs nés en Europe ont pour la majorité (6/10) une épouse de même origine qu'eux, trois ont épousé une Québécoise et l'un a une épouse algérienne. Ce sont surtout des ménages d'origine française (6 de France et 1 de Belgique), à l'exception des deux ménages norvégiens et d'un ménage irlandais. Si l'on considère l'ensemble des 15 ménages et quel que soit le lieu de naissance de l'épouse, $40 \%$ ne comprennent que des enfants déclarés nés au Québec.

Au recensement de 1881, 24 chefs de ménage sont nés aux États-Unis ou en Europe. Trois sont nés aux États-Unis, avec respectivement une épouse née aux États-Unis, une née en Écosse et une née au Québec. Ils ont 40 ans ou moins. Les 21 autres chefs de ménage sont européens et ont en moyenne 45,2 ans. Quatorze d'entre eux ont une épouse de même origine qu'eux. Un seul a épousé une Québécoise. Six sont célibataires ou veufs. La distribution spatiale des ménages en 1881 est plus parlante que celle des ménages au recensement de 1891, car les ménages sont un peu plus nombreux. Les regroupements selon le pays de naissance sont évidents, les Français présentant un premier regroupement, les anglophones (Anglais, Écossais, Irlandais), plus nombreux, en forment un second. D'ailleurs, selon l'Annuaire du Séminaire (1897-1898), plusieurs de ces derniers se sont établis sur le rang Nouvelle-Angleterre. Si l'on considère l'ensemble des 24 ménages et quel que soit le lieu de naissance de l'épouse, $29 \%$ ne comprennent que des enfants déclarés nés aux Québec.

\section{Ménages dont le chef est né au Québec}

En 1891, dans la plupart des 137 ménages (tableau 7) dont le chef est né au Québec, l'épouse est également née au Québec. Cette combinaison représente $83 \%(114 / 137)$ des ménages. Près de la moitié $(48 \%)$ de ces derniers ont tous leurs enfants recensés déclarés nés au Québec et plus du tiers

9. Selon les instructions, le recenseur devait faire l'énumération des ménages en attribuant un numéro séquentiel en fonction de l'ordre de visite des maisons habitées et des «familles » qui y résidaient. Il est légitime de faire l'hypothèse que le recenseur, par économie de temps, a suivi un parcours qui respectait l'ordre des voisinages et que la séquence des numéros de ménage correspond à cet ordre. 
(37\%) sont des ménages dont les enfants sont nés aux États-Unis ou en alternance Québec-États-Unis. Enfin, une bonne proportion (15\%) des ménages n'a pas d'enfant recensé. Les ménages dont l'épouse est née aux États-Unis sont très peu nombreux (7/137, soit $5 \%)$, de même que ceux dirigés par un veuf ou une veuve. Ils présentent diverses combinaisons de lieu de naissance des enfants. Au total, parmi les chefs de ménage nés au Québec, ceux qui ont fait un séjour aux États-Unis représentent un pourcentage relativement important: en effet, 48 ménages, soit $35 \%$, déclarent au moins une naissance d'enfant aux États-Unis. Si l'on ajoute les couples dont l'épouse est née aux États-Unis, le total s'élève à $38 \%$. Le recensement sous-estime probablement ce groupe. En 1881, les données sont très similaires à celles de 1891, avec une majorité de ménages dont le chef et sa conjointe sont nés au Québec: $86 \%(112 / 130)$ et $83 \%$ en 1891. Environ $60 \%$ des ménages avec enfants ont tous leurs enfants déclarés nés au Québec (69/115) et $57 \%(63 / 111)$ en 1891. Ce pourcentage pourrait être surestimé alors que le nombre de ménages (46/130) identifiés comme ayant séjourné aux États-Unis (35\%) est probablement sous-estimé tout comme en $1891(38 \%)$.

TABLEAU 7 Distribution des ménages recensés en 1891 et en 1881 à La Patrie dont le chef est né au Québec, selon le lieu de naissance de l'épouse et selon celui des enfants

\begin{tabular}{|l|c|c|c|c|}
\hline Nombre de ménages & $\begin{array}{c}\text { Enfants nés } \\
\text { au Québec }\end{array}$ & $\begin{array}{r}\text { Enfants nés aux É.-U. ou } \\
\text { en alternance Québec-É.-U. }\end{array}$ & $\begin{array}{c}\text { Sans enfant } \\
\text { recensé }\end{array}$ & Total \\
\hline $\mathbf{1 8 9 1}$ & \multicolumn{5}{|l|}{} \\
\hline Épouse née au Québec & 55 & 42 & 17 & 114 \\
\hline Épouse née aux É.-U. & 4 & 3 & 0 & 7 \\
\hline Veuf, veuve, séparé & 4 & 3 & 2 & 9 \\
\hline Célibataire & 0 & 0 & 7 & 7 \\
\hline Total & $\mathbf{6 3}$ & $\mathbf{4 8}$ & $\mathbf{2 6}$ & $\mathbf{1 3 7}$ \\
\hline $\mathbf{1 8 8 1}$ & & \multicolumn{4}{|c|}{} \\
\hline Épouse née au Québec & 64 & $39^{\mathrm{b}}$ & 9 & 112 \\
\hline Épouse née aux É.-U. & $1^{\mathrm{a}}$ & 5 & 0 & $6^{\mathrm{a}}$ \\
\hline Veuf, veuve, séparé & 4 & 2 & 0 & 6 \\
\hline Célibataire & 0 & 0 & 6 & 0 \\
\hline Total & $\mathbf{6 9}$ & $\mathbf{4 6}$ & $\mathbf{1 5}$ & $\mathbf{1 3 0}$ \\
\hline
\end{tabular}

a un ménage avec l'épouse née en Norvège

${ }^{b}$ un ménage avec 3 des enfants nés en Ontario 
Nous avons observé un certain regroupement des ménages ayant fait un séjour aux États-Unis (enfants ou conjoint nés aux États-Unis). Les noms des rangs en 1875 (Comité du centenaire de La Patrie, 1975; Annuaire du Séminaire, 1997-1998 et 1898-1899) confirment l'existence de regroupements des ménages selon leur provenance, et même selon l'appartenance à des familles spécifiques : rangs Petite Angleterre, Petit Canada, Petit Québec, Cohoes, Dubreuil, Labonne... Cependant, tant que les familles franco-américaines n'auront pas toutes été identifiées à l'aide d'autres sources et d'autres approches, leur localisation par rapport aux familles originaires du Québec ne sera pas entièrement fiable.

\section{Parcours migratoire des ménages}

Le départage des ménages selon l'origine franco-américaine, européenne et québécoise a mis en évidence des mouvements d'allers-retours transfrontaliers, en réponse à des stratégies familiales dans un contexte économique continuellement changeant.

Les ménages recensés ont été répartis en 4 grands groupes déterminés par les lieux de naissance des enfants : (1) tous les enfants sont nés au Québec; (2) tous les enfants sont nés aux États-Unis; (3) une partie des enfants est née en Europe; (4) les naissances se font en alternance aux États-Unis et au Québec dans diverses combinaisons. Un $5^{\mathrm{e}}$ groupe comprend les ménages sans enfant. Par définition, les groupes 2 et 4 sont constitués de ménages franco-américains puisqu'au moins un membre du ménage est né aux États-Unis.

Les ménages dont tous les enfants sont nés au Québec, tant en 1891 qu'en 1881 (tableau 8), comptent pour un peu moins de la moitié des ménages ( $45 \%$ et $49 \%$ ), alors que ceux dont tous les enfants sont nés aux États-Unis sont rares, quoique plus nombreux en 1891 qu'en 1881 (5\% et $1 \%)$. Les ménages dont un ou plusieurs enfants sont nés en Europe représentent respectivement $2 \%$ et $7 \%$ des ménages. Les ménages qui ont fait un ou plusieurs allers-retours Québec-États-Unis représentent près du tiers des ménages ( $29 \%$ et $30 \%$ ). Enfin, les ménages sans enfant sont un peu plus nombreux en 1891 qu'en 1881 (19\% et $13 \%)$.

Chaque groupe a ensuite été subdivisé selon l'âge des chefs de ménage afin de relativiser l'information contenue dans le ménage, puisqu'un jeune chef de ménage ou un chef âgé aura par définition peu d'enfants recensés avec lui. Les ménages dont le chef a un âge intermédiaire devraient avoir davantage d'enfants recensés, ce qui permet de mettre en évidence les trajets migratoires (tableau 9). 
TABLEAU 8 Distribution des ménages aux recensements de 1891 et de 1881 selon les lieux de naissance des enfants

\begin{tabular}{|c|c|c|c|c|}
\hline \multirow[t]{2}{*}{ Types de ménage } & \multicolumn{2}{|c|}{1891} & \multicolumn{2}{|c|}{1881} \\
\hline & Ensemble & $\begin{array}{l}\text { Parents nés } \\
\text { au Québec }^{\mathrm{a}}\end{array}$ & Ensemble & $\begin{array}{l}\text { Parents nés } \\
\text { au Québec }\end{array}$ \\
\hline (1) Enfants tous nés au Québec & 69 & 59 & 75 & 67 \\
\hline (2) Enfants tous nés aux États-Unis & 7 & 6 & 1 & 1 \\
\hline (3) Des enfants nés en Europe & 3 & o & 11 & o \\
\hline $\begin{array}{l}\text { (4-1) Enfants nés au Québec } \\
\text { puis aux É.-U. }\end{array}$ & 10 & 10 & 14 & 13 \\
\hline $\begin{array}{l}\text { (4-2) Enfants nés aux É.-U. } \\
\text { puis au Québec }\end{array}$ & 24 & 20 & 25 & 21 \\
\hline $\begin{array}{l}\text { (4-3) Enfants nés au Québec, É.-U., } \\
\text { Québec }\end{array}$ & 10 & 9 & 8 & 6 \\
\hline (5) Ménages sans enfants & 29 & 27 & 20 & 16 \\
\hline Total & 152 & 131 & 154 & 124 \\
\hline
\end{tabular}

a Ménages dont le parent (un veuf ou une veuve) ou les parents (un couple) sont nés au Québec ou, pour les ménages sans enfant, ménages dont tous les membres recensés sont nés au Québec.

Pour chacune des catégories ainsi créées, les écarts d'âge entre les époux, l'âge des conjoints à la naissance du $1^{\text {er }}$ enfant recensé et les intervalles de naissance entre les enfants ont été examinés (annexe A), afin de vérifier si certains ménages ne pourraient pas ultérieurement être qualifiés comme franco-américains ou si encore les allers-retours transfrontaliers ne pouvaient pas être mieux documentés. La prise en compte de ces éléments pour chacune des catégories migratoires basées sur les lieux de naissance des enfants du ménage suggère beaucoup de scénarii explicatifs. Sans les jumelages entre recensements et les jumelages avec les diverses données d'état civil et la reconstitution de généalogies ascendantes sur une ou deux générations, il est présentement impossible d'adopter un scénario plutôt qu'un autre. Il faut se contenter de quantifier les situations (tableau 9).

\section{Ménages dont tous les enfants sont nés au Québec}

La majorité des ménages dont tous les enfants sont nés au Québec est composée, tant en 1891 (59/69) qu'en 1881 (67/75), de couples dont les deux conjoints sont aussi nés au Québec. Ce sont les 30-39 ans qui sont les plus représentés en 1891, suivis en proportion à peu près égale des autres groupes d'âge à l'exception des 60 ans et plus. En 1881, les groupes sont un 


\begin{tabular}{|c|c|c|c|c|c|c|c|c|c|c|c|c|c|c|c|c|}
\hline & 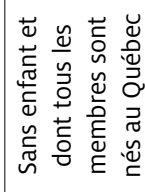 & $\stackrel{m}{\sim}$ & $m$ & $m$ & $N$ & 0 & $\hat{\mathbf{N}}$ & 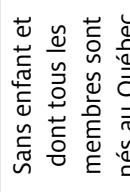 & $m$ & 6 & $m$ & $N$ & - & - & 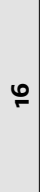 & 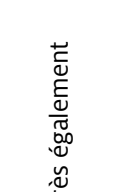 \\
\hline & 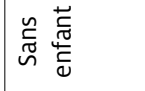 & $m$ & $m$ & $m$ & $N$ & $\infty$ & ก & 点 & $m$ & 0 & $\nabla$ & $N$ & $m$ & $N$ & i & $\underset{\substack{0 \\
. \pi}}{\vec{\alpha}}$ \\
\hline & 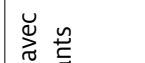 & $\Xi$ & $\hat{m}$ & $\rho$ & $\stackrel{\rho}{\sigma}$ & $\stackrel{n}{\sim}$ & $\stackrel{\Xi}{\circ}$ & 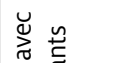 & $\circ$ & $\stackrel{\stackrel{n}{N}}{2}$ & $\bar{m}$ & $\tilde{\lambda}$ & ন & 0 & $\begin{array}{l}\infty \\
0 \\
0\end{array}$ & \\
\hline & $\stackrel{\frac{\pi}{0}}{\frac{E}{0}}$ & 9 & 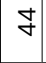 & $\ddot{\sim}$ & ৩ & $\neq$ & 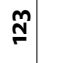 & $\stackrel{\pi}{\circ}$ & $\circ$ & $\hat{N}$ & $\overline{7}$ & $\bar{m}$ & $\stackrel{\infty}{\sim}$ & $N$ & $\stackrel{ \pm}{m}$ & 选 \\
\hline & 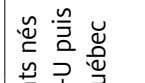 & ชู & $\stackrel{0}{\circ}$ & $m$ & 0 & $m$ & ำ & 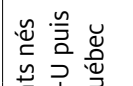 & 0 & $m$ & $m$ & $N$ & $N$ & - & $\overline{\boldsymbol{N}}$ & 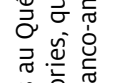 \\
\hline & 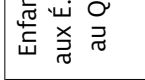 & in & $\stackrel{\rho}{\sim}$ & in & 0 & $m$ & $\mathbb{N}$ & 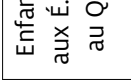 & 0 & $\nabla$ & $\stackrel{n}{\sim}$ & $m$ & $N$ & - & $\stackrel{\boldsymbol{n}}{\boldsymbol{n}}$ & 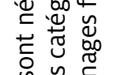 \\
\hline & صِّ & 0 & $\nabla$ & $\stackrel{\pi}{m}$ & $N$ & 0 & $\sigma$ & 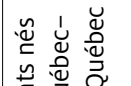 & 0 & $N$ & - & $N$ & - & 0 & 0 & 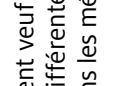 \\
\hline $\bar{\sigma}$ & 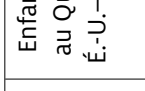 & 0 & in & $\stackrel{\pi}{m}$ & $N$ & 0 & $\stackrel{0}{2}$ & نُ. & 0 & $N$ & - & $\nabla$ & $r$ & 0 & $\infty$ & 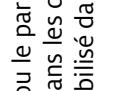 \\
\hline 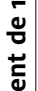 & 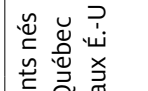 & - & 0 & - & $m$ & in & $\circ$ & 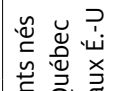 & 0 & 0 & $N$ & $m$ & $\lambda$ & - & $\underline{m}$ & 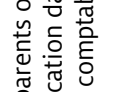 \\
\hline 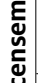 & 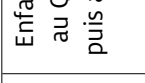 & - & 0 & - & $m$ & in & $\circ$ & 立 & 0 & 0 & $m$ & $m$ & $\Lambda$ & - & $\Xi$ & 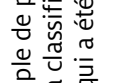 \\
\hline ๘ & 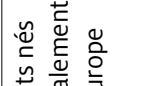 & 0 & 0 & 0 & 0 & 0 & 0. & 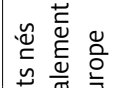 & 0 & 0 & 0 & 0 & 0 & 0 & 0 & 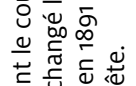 \\
\hline & 恖 & 0 & - & 0 & 0 & $N$ & $m$ & 曽产妾 & 0 & 0 & $N$ & Ln & $m$ & - & $F$ & 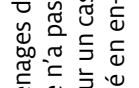 \\
\hline & ن & - & 0 & - & n & N & 0 & ن. & 0 & o & 0 & o & - & 0 & - & 5 \\
\hline & 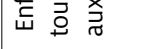 & - & - & - & $N$ & $N$ & $\Lambda$ & 岌 & 0 & 0 & 0 & o & - & 0 & - & 00 \\
\hline & 党 & $\infty$ & $\stackrel{\sim}{N}$ & $=$ & $\simeq$ & in & กุ่ & 营 & 0 & ㅇ & 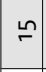 & $\stackrel{\sim}{\curvearrowleft}$ & $m$ & $\nabla$ & $\hat{\sigma}$ & ע \\
\hline & Ш匚 & $\simeq$ & $\varphi_{1}$ & $m$ & $m$ & in & 8 & 山匚 & 0 & $\bar{N}$ & ก & $\stackrel{\varphi}{r}$ & $\checkmark$ & $\nabla$ & ก & 气े \\
\hline & $\frac{y}{n} \underset{\tilde{c}}{\stackrel{a}{\sigma}}$ & $\hat{N}$ & f & $\boldsymbol{N}$ & $\bar{N}$ & $\overline{\boldsymbol{N}}$ & $\bar{m}$ & $\underline{y} \underset{\tilde{\sigma}}{\tilde{u}}$ & $m$ & $\bar{m}$ & $\stackrel{+}{m}$ & $\mathbb{N}$ & $\stackrel{\stackrel{n}{\boldsymbol{N}}}{ }$ & $N$ & 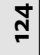 & \\
\hline & $\bar{\circ} \cdot \bar{\Xi}$ & $\tilde{m}$ & 于 & $\stackrel{N}{N}$ & N & $\stackrel{\boldsymbol{N}}{\boldsymbol{N}}$ & $\tilde{n}$ & 온 & $m$ & $m$ & ห & $\dot{m}$ & $\stackrel{\circ}{m}$ & $a$ & 亡ே & 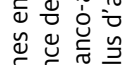 \\
\hline & 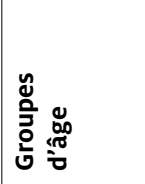 & 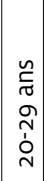 & $\mid \begin{array}{c}n \\
\tilde{c} \\
0 \\
2 \\
m \\
0 \\
m\end{array}$ & $\begin{array}{l}n \\
\tilde{\sigma} \\
o \\
q \\
\dot{\gamma} \\
\dot{y}\end{array}$ & $\begin{array}{l}u \\
\tilde{c} \\
o \\
\hat{n} \\
0 \\
\text { กิ }\end{array}$ & \begin{tabular}{l|l}
$\frac{n}{3}$ \\
0 \\
+ \\
0 \\
$气$ \\
0 \\
0 \\
0
\end{tabular} & $\begin{array}{l}\mathbf{k} \\
\mathbf{5} \\
\mathbf{1}\end{array}$ & 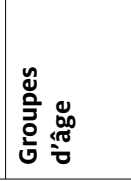 & 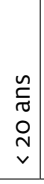 & 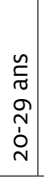 & $\mid \begin{array}{l}n \\
\tilde{c} \\
0 \\
0 \\
2 \\
0 \\
0 \\
m\end{array}$ & 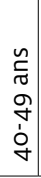 & 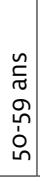 & 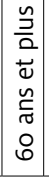 & 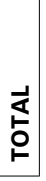 & 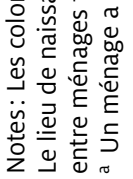 \\
\hline
\end{tabular}


peu plus équilibrés, décroissant de façon peu prononcée des 20-29 ans aux 50-59 ans.

L'analyse des écarts d'âge (annexe A) entre les membres du ménage révèle que, en 1891, dans chacun des groupes d'âge, un certain nombre de couples sont susceptibles d'avoir eu des enfants dont les naissances échappent à l'observation et pour lesquels on ne peut certifier qu'ils soient nés au Québec. Des écarts d'âge importants entre les enfants recensés, entre les époux, ou des écarts d'âge trop courts entre la mère et les aînés des enfants laissent entrevoir la possibilité que des enfants issus de deux lits cohabitent et que certains enfants du premier lit aient déjà quitté le ménage. Chez les couples plus âgés qui n'ont plus que deux ou trois enfants relativement jeunes, on peut aussi penser que les aînés sont partis. Au total, 15 couples sont susceptibles d'avoir fait des séjours hors de la communauté. Leur distribution ne suit pas celle de l'ensemble de cette catégorie : plus de la moitié sont des ménages dont le chef a 50 ans ou plus. Sur les 75 ménages dont tous les enfants recensés en 1881 sont nés au Québec, 17 d'entre eux présentent aussi des écarts d'âge qui suggèrent que des enfants échappent à l'observation, particulièrement chez les 50 ans et plus.

\section{Ménages dont tous les enfants sont nés aux États-Unis ou en Europe}

Il n'y a que 7 ménages en 1891 et 1 en 1881 dont tous les enfants sont nés aux États-Unis. Les ménages dont des enfants sont nés en Europe sont peu nombreux, surtout en 1891; un de ces ménages a eu des enfants aux ÉtatsUnis. L'examen des écarts d'âge montre qu'un certain nombre de ménages dans le groupe d'âge des 50 ans et plus ont pu avoir d'autres enfants aux États-Unis ou au Québec.

\section{Ménages avec diverses combinaisons d'aller-retour Québec-États-Unis}

En 1891, sur les 44 ménages dont les enfants recensés sont nés au Québec et aux États-Unis selon diverses combinaisons d'alternance des lieux de naissance, 10 ménages ont eu des enfants au Québec puis aux États-Unis. Ce sont majoritairement des couples de 50 ans ou plus dont les familles sont déjà complétées lors du recensement. Dix ménages dont les enfants sont nés au Québec puis aux États-Unis puis au Québec sont d'âge intermédiaire (30-49 ans) et ont fait de brefs séjours aux États-Unis, puisque seulement un ou deux enfants y sont nés. Les 24 ménages dont les enfants sont déclarés nés aux États-Unis puis au Québec appartiennent surtout au groupe d'âge des 30-39 ans. Il s'agit probablement de couples formés aux 
États-Unis. La majorité d'entre eux a eu plus d'enfants au Québec qu'aux États-Unis. Au total, sur les 44 ménages ayant fait des allers-retours, 5 d'entre eux, appartenant au groupe des 60 ans et plus, sont susceptibles d'avoir séjourné hors Québec sans que ce séjour soit marqué par une naissance d'enfant recensé à La Patrie. Pour les 39 autres ménages, les intervalles entre les naissances des enfants et les écarts d'âge entre les parents et les enfants ne laissent pas de place pour cette éventualité et ces ménages seraient donc bien classés.

Sur les 47 ménages de 1881 présentant diverses combinaisons d'allerretour, 14 ménages ont des enfants nés au Québec puis aux États-Unis et plus de la moitié des chefs ont 50 ans et plus. Quant aux 8 familles qui ont fait des allers-retours Québec-États-Unis-Québec, la moitié de ces couples est dans le groupe des 40-49 ans. Vingt-cinq ménages ont eu des enfants aux États-Unis puis au Québec et $76 \%$ des chefs de ce groupe ont moins de 40 ans. Au total, 19 des 47 ménages sont susceptibles d'avoir fait des allers-retours qui échappent à l'observation. Contrairement à ce qu'on a observé jusqu'à maintenant, ils se distribuent dans tous les groupes d'âge à l'exception des 20-29 ans, mais se concentrent chez les 50-59 ans.

\section{Les ménages sans enfant}

Les 29 ménages qui sont recensés sans enfant en 1891 sont surtout des couples mariés (20); on compte aussi 7 célibataires, un jeune veuf et un homme séparé. Ce sont surtout de très jeunes ménages (45\%), composés de célibataires ou de jeunes couples qui n'ont pas encore fondé leur famille, ou bien des ménages âgés ( $28 \%$ ) dont les enfants ont quitté le foyer pour s'établir. Ces derniers sont susceptibles d'avoir séjourné aux États-Unis. Les 20 ménages sans enfant recensé en 1881 comprennent 8 ménages de célibataires, 2 veufs, 5 jeunes couples qui n'ont pas eu le temps d'avoir des enfants et 5 couples plus âgés pour lesquels un séjour aux États-Unis est envisageable.

\section{Déclaration du lieu de naissance des parents de l'individu recensé}

Le recensement de 1891 renseigne sur le lieu de naissance des parents de chaque individu recensé. Évidemment, pour chaque enfant du ménage, les lieux de naissance de son père et de sa mère sont redondants avec l'information donnée pour le parent quant au pays ou à la province de naissance. Cependant, dans le cas des veufs et veuves avec enfants, cette information permet de connaître le lieu de naissance du conjoint décédé. Cet examen 
n’a pas modifié le départage initial des ménages franco-américains, puisque le lieu de naissance du parent décédé s'est révélé être le Québec.

\section{Bilan}

La catégorisation des ménages basée sur les seules déclarations du lieu de naissance de ses membres évalue minimalement à $38 \%$ en 1891 et $32 \%$ en 1881 la fréquence des ménages franco-américains. L'examen des écarts d'âge estime que $21 \%$ (1891) et $28 \%$ (1881) des ménages devront faire l'objet d'une recherche plus approfondie par des approches et des sources complémentaires afin de départager plus adéquatement les familles en provenance des États américains de celles qui seraient venues directement des paroisses et des villes du Québec. La majorité se retrouve dans le groupe d'âge des 50 ans et plus. Il faudra attendre la fin de l'enquête pour interpréter cette information : nous serons alors en mesure de mieux estimer le nombre de familles qui ont été effectivement rapatriées, que ce soit par un recrutement des sociétés de colonisation ou encore suite à la promulgation de l'Acte de rapatriement de 1875.

\section{LES DÉCLARATIONS DE PROFESSION}

Le dépouillement des déclarations individuelles de profession et le calcul des fréquences sont des opérations fastidieuses, mais elles permettent de caractériser les activités économiques et de les comparer afin d'observer, le cas échéant, les modifications dans les structures sociales. Plus important ici, la caractérisation des ménages selon les activités déclarées par ses membres documente adéquatement la façon dont la famille s'organise et s'ajuste aux changements.

\section{Les déclarations individuelles}

Il est normal que plusieurs professions soient communes aux deux recensements, alors que d'autres sont spécifiques de l'un ou de l'autre (annexe B). La plus grande variété des professions en 1891 illustre le développement de la communauté, ce dont témoigne également l'apparition de déclarations de profession pour des femmes.

Deux difficultés sont inhérentes au recensement de 1881, et elles concernent toutes deux la déclaration du métier de cultivateur. Premièrement, lors du dépouillement du recensement de 1881 par les mormons, la profession «cultivateur » inscrite par le recenseur francophone dans la 
colonne dédiée à cette variable a été traduite par le mot anglais farmer. Or les deux termes reflètent une réalité fort différente : en français, le cultivateur est celui qui possède la terre et est son propre patron, alors que le fermier est un employé (habituellement sous contrat appelé bail à ferme). À La Patrie, cette distinction entre fermier et cultivateur a été faite par le recenseur, et la vérification sur la copie du manuscrit a permis de corriger, pour nos analyses, cette erreur systématique de traduction. Erreur mal venue, puisqu'en théorie la saisie, plus de cent ans plus tard, aurait dû être faite en respectant le contexte linguistique ${ }^{10}$. Deuxièmement, le commissaire du recensement a biffé la déclaration "cultivateur » inscrite initialement par le recenseur pour tous les fils du ménage de moins de 21 ans exerçant cette activité et l'a remplacé par le mot anglais "son» placé au-dessus de la biffure ${ }^{11}$. Cette «nouvelle» profession, transcrite farmer's son (fils de cultivateur) par les mormons, a été incluse pour nos analyses dans un groupe à part. La création de cette catégorie par le commissaire, à partir de l'âge déclaré lors du recensement, produit à l'occasion des « fils de cultivateur " qui n'en sont visiblement pas : ils ont, à peu près, le même âge que le chef de ménage. Il s'agit fort probablement de frères, surtout lorsqu'ils portent le même patronyme.

En 1891, le métier de cultivateur regroupe majoritairement des chefs et leurs fils, alors qu'en 1881, il faut ajouter à ce compte la déclaration de farmer's son. La profession de cultivateur demeure la plus fréquemment déclarée aux deux dates (annexe B) : elle représente $67 \%$ des déclarations en 1891 et $80 \%$ en 1881 . Un plus grand nombre de fils exercent ce métier en 1881, ce qui ne surprend pas étant donné que cette période suit de très près la période initiale d'établissement des familles, période où de nombreux bras sont requis et où les métiers reliés à d'autres activités commencent à peine à faire leur apparition. Sur l'ensemble des 229 déclarations de profession en 1891, les activités rattachées au travail agricole représentent $72 \%$

10. Bibliothèque et archives Canada (http ://www.collectionscanada.gc.ca/base-dedonnees/recensement-1881/001049-3000-f.html) indique, à tort, que le contenu de la basse de données respecte la langue d'origine utilisée dans les documents et que ces informations n'ont pas été traduites.

11. En dépit des instructions aux recenseurs, qui étaient pourtant bien claires : «Les enfants qui suivent la profession de leur père ou lui sont associés doivent porter la même indication. Par exemple, un fils de cultivateur travaillant avec son père doit être désigné comme "cultivateur", le fils d'un charpentier comme "charpentier". " Le texte anglais est tout aussi clair: "When sons follow the professions or occupations of their fathers, and are associated with them, the same description is to be inserted. For instance, a farmer's son working on his father's farm, is a farmer; a carpenter's son, in the same way, a carpenter » (Département de l'Agriculture, 1881). 
(165/229). Les autres professions sont, par ordre décroissant d'importance, forestier, institutrice, rentier ou rentière, fileuse, marchand et menuisier. Quelques individus exercent d'autres métiers. Les professions reliées au travail agricole sont aussi les plus représentées en 1881 (222/260, soit $85 \%$ ), suivies de loin par celle de journalier (11/260). Aucune femme ne déclare de profession sauf 4 institutrices, alors qu'en 1891, 9 femmes déclarent exercer une profession.

L'importance et le rôle de l'industrie forestière dans les zones de colonisation sont un fait connu, étudié et débattu (voir entre autres Séguin, 1977; Bouchard, 1977, 1978) et La Patrie ne fait pas exception. Dans ce contexte, la faible représentation, aux deux recensements, des activités liées à l'industrie du bois surprend, d'autant plus que Little (1977) fait état de l'importance de la forêt dans la survie des familles au début de la colonisation, et que l'ethnologue P. Nadon (1970) spécifie que pour toutes les périodes de l'histoire de la communauté " pour celui qui n'a que sa ferme, sans le soutien d'aucune activité complémentaire (bois, porcherie, érablière, etc.), il est pratiquement impossible de survivre. [...] le bois, parce que tous en possèdent, se révèle le principal complément à l'agriculture ». Nadon ajoute que le cultivateur devient souvent bûcheron durant une partie de l'année afin de gagner quelque argent, et que les scieries procurent un revenu secondaire aux cultivateurs qui s'y emploient. On peut aussi spéculer que certains journaliers ont pu être employés dans les moulins à bois. Le Registre de la population (Mayer, 1977) qui donne la profession du père au baptême de chacun des enfants montre une variation dans les déclarations selon les saisons, et plus souvent l'alternance cultivateur/bûcheron. La date du recensement a pu avoir introduit un certain biais, mais il est plus probable que ni les recensés, ni le recenseur n'ont jugé bon de rapporter dans un document officiel cette activité, considérée comme d'appoint. Les instructions aux recenseurs, tant en 1881 qu'en 1891, laissent une certaine souplesse dans l'inscription des professions : elles précisent que «quand deux professions sont pratiquées par la même personne, on peut les inscrire toutes les deux, ou n'en inscrire qu'une selon l'avis de la personne elle-même ». La présence de 9 forestiers en 1891 témoigne cependant d'une certaine spécialisation professionnelle liée à l'activité des scieries.

\section{Les déclarations de profession par ménage}

Les déclarations individuelles de profession montrent des changements dans la structure occupationnelle de la communauté entre les deux recen- 
sements. Les facteurs de reproduction familiale et sociale qui l'alimentent peuvent être documentés, du moins en partie, par l'examen des professions du chef du ménage en lien avec les métiers exercés par les autres membres de l'unité. La prise en compte de la provenance des familles peut révéler des pratiques différentes quant aux activités déclarées et à leur transmission. Pour les analyses, nous avons distingué deux groupes : celui des «cultivateurs", qui comprend les ménages dont les déclarations de métier de ses membres, y compris le chef de ménage, sont exclusivement celles de cultivateur, et celui des « autres métiers » catégorie qui regroupe les ménages dont les membres déclarent d'autres métiers que cultivateur, sans exclure toutefois ce dernier. Chacune de ces catégories a par la suite été subdivisée.

Toutes origines confondues (figure 1), les ménages de cultivateurs sont les plus nombreux en 1891 (67\%) comme en 1881 (78\%), avec cependant une perte de $11 \%$ due à l'augmentation de métiers non agricoles. En effet, en 1891, $33 \%$ des ménages n'étaient pas exclusivement dans l'agriculture, alors que le recensement de 1881 ne comptait que $22 \%$ de ménages dans cette catégorie. Cette augmentation, qui se fait au bénéfice des ménages québécois, est cohérente avec la diversification professionnelle qui accompagne le changement de statut de colonie à celui de communauté établie. Le fait que ce soient les ménages originaires du Québec qui se qualifient

FIGURE 1 Distribution des ménages selon les déclarations de profession et selon la provenance aux recensements de La Patrie en 1891 et en 1881

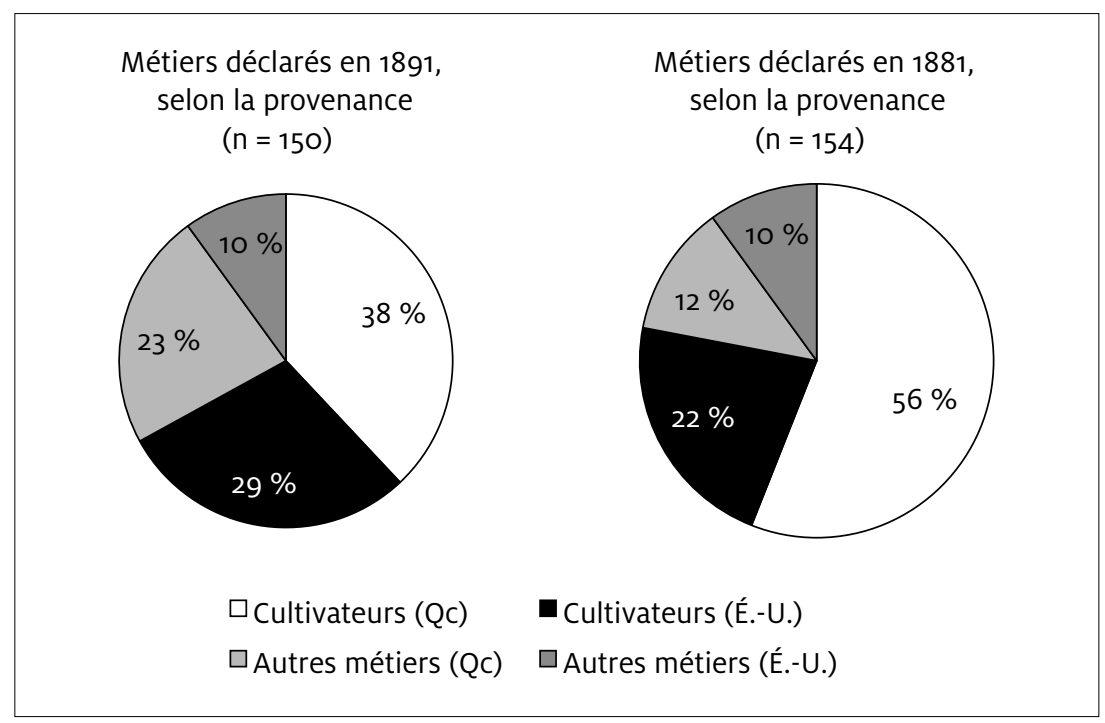


dans cette catégorie est à souligner. Les ménages de cultivateurs provenant du Québec représentent $38 \%$ des unités recensées en 1891, alors qu'ils étaient en plus forte proportion ( $56 \%$ ) dix ans plus tôt, ce qui témoigne d'un éloignement de la profession au cours de la période intercensitaire. $\mathrm{La}$ représentation des ménages de cultivateurs franco-américains a peu varié en dix ans, avec un peu plus et un peu moins du quart des ménages dans cette catégorie (respectivement $29 \%$ et $22 \%$ ).

La question de la reproduction et de la diversification des professions comme stratégies d'ajustement de la famille et du ménage aux conjonctures économiques est traitée ici par le biais de la création, annoncée plus haut, de quatre types de ménage (tableau 10). Les ménages québécois dont plus d'un membre déclare gagner sa vie dans l'agriculture (type 1) sont moins nombreux en 1891 qu'en 1881 (19\% contre $27 \%$ ) et en moyenne plus âgés (54 ans contre 42 ans), probablement parce qu'une partie des premières familles immigrantes est demeurée dans la communauté. Ces familles ont cependant encore des fils et parfois d'autres membres (père, beau-père ou frère) qui participent à l'entreprise familiale. Les ménages dont la seule profession déclarée est celle du chef cultivateur (type 2) sont les plus nombreux, leur chef est en moyenne plus jeune ( 38 ans et 35 ans), et ce sont plus rarement des ménages de célibataires ou de couples qui n'ont pas encore de fils, ou dont les fils ne sont pas encore en âge de déclarer une profession. On peut penser qu'un certain nombre de ces chefs étaient déjà cultivateurs chez leur père en 1881 et qu'ils se sont établis sur une exploitation agricole au cours de la période intercensitaire. Plusieurs interprétations de ces chiffres sont envisageables, mais les effectifs sont peu nombreux et les explications peuvent relever du type de migration comme de la stabilité de certaines familles de cultivateurs, sans que l'on puisse trancher en faveur de l'une plutôt que de l'autre. Les ménages franco-américains dont le chef et les autres membres déclarent la profession de cultivateur exclusivement (type 1) sont proportionnellement plus nombreux (26\% et $38 \%$ ) que les ménages originaires du Québec (19\% et $27 \%$ ) et leurs chefs sont aussi plus âgés (56 ans contre 51 ans), alors que les ménages franco-américains dont la seule profession déclarée est celle du chef de ménage cultivateur (type 2), sont dans la trentaine et demeurent la catégorie prédominante $(74 \%$ et $62 \%)$, ce qui est également le cas chez les cultivateurs québécois de cette même catégorie ( $81 \%$ et $73 \%$ ). Le nombre plus important en 1881 de ménages québécois et franco-américains comprenant plus d'un membre cultivateur (type 1) est cohérent avec la réalité d'une immigration récente de familles ayant besoin de plusieurs paires de bras pour aider à la préparation et à l'exploitation des lots octroyés. 
TABLEAU 10 Distribution des ménages aux recensements de la Patrie en 1891 et en 1881 selon les catégories et les types de profession, ainsi que selon la provenance

\begin{tabular}{|c|c|c|c|c|}
\hline \multicolumn{2}{|l|}{ Professions } & \multirow{2}{*}{$\begin{array}{c}\text { Provenance } \\
\text { Québec }^{\mathrm{a}}\end{array}$} & \multirow{2}{*}{$\begin{array}{c}\mathbf{1 8 9 1} \\
\begin{array}{c}\text { Nombre de ménages } \\
\% ; \\
\text {; âge moyen du chef } \\
\text { de ménage }\end{array} \\
58 \\
19 \% ; 54 \text { ans } \\
81 \% ; 38 \text { ans }\end{array}$} & \multirow{2}{*}{$\begin{array}{c}\mathbf{1 8 8 1} \\
\begin{array}{c}\text { Nombre de ménages } \\
\text { \%; âge moyen du } \\
\text { chef de ménage }\end{array} \\
86 \\
27 \% ; 42 \text { ans } \\
73 \% ; 35 \text { ans }\end{array}$} \\
\hline \multirow{2}{*}{ CULTIVATEURS } & $\begin{array}{l}\text { Type } 1 \\
\text { Type } 2\end{array}$ & & & \\
\hline & $\begin{array}{l}\text { Type } 1 \\
\text { Type } 2\end{array}$ & États-Unis & $\begin{array}{c}43 \\
26 \% ; 56 \text { ans } \\
74 \% ; 36 \text { ans }\end{array}$ & $\begin{array}{c}34 \\
38 \% ; 51 \text { ans } \\
62 \% ; 38 \text { ans }\end{array}$ \\
\hline Sous-total & & & 101 & 120 \\
\hline \multirow{2}{*}{ AUTRES MÉTIERS } & $\begin{array}{l}\text { Type } 3 \\
\text { Type } 4\end{array}$ & Québec ${ }^{\mathrm{a}}$ & $\begin{array}{c}34 \\
29 \% ; 53 \text { ans } \\
71 \% ; 40 \text { ans }\end{array}$ & $\begin{array}{c}19 \\
26 \% ; 45 \text { ans } \\
74 \% ; 43 \text { ans }\end{array}$ \\
\hline & $\begin{array}{l}\text { Type } 3 \\
\text { Type } 4\end{array}$ & États-Unis & $\begin{array}{c}15 \\
27 \% ; 53 \text { ans } \\
73 \% ; 43 \text { ans }\end{array}$ & $\begin{array}{c}15 \\
40 \% ; 55 \text { ans } \\
60 \% ; 38 \text { ans }\end{array}$ \\
\hline Sous-total & & & 49 & 34 \\
\hline Total & & & $150^{\mathrm{b}}$ & 154 \\
\hline
\end{tabular}

Type 1: tous les membres du ménage qui déclarent une profession sont cultivateurs; Type 2: la seule déclaration de profession est celle du chef de ménage cultivateur; Type $\mathbf{3}$ : le chef de ménage est cultivateur mais il peut déclarer aussi un autre métier, ou bien d'autres membres du ménage exercent un autre métier que celui de cultivateur; Type $\mathbf{4}$ : le chef de ménage déclare une profession autre que celle de cultivateur et les autres membres peuvent être cultivateurs ou exercer un autre métier.

a Les ménages originaires du Québec, d'Europe et ceux sans enfant déclaré ont été regroupés afin de prévenir la création de catégories comprenant de trop petits effectifs, les deux derniers groupes étant très peu nombreux.

b Deux ménages exclus, aucun des membres du ménage n’ayant déclaré de profession.

La catégorie «autres métiers » comprend, d'une part, des ménages dont le chef est cultivateur et exerce lui-même un autre métier ou encore dont certains des fils, filles ou autres membres déclarent une profession " autre » (type 3) et, d'autre part, des ménages dont le chef exerce lui-même une autre profession que celle de cultivateur, combinée ou non à un deuxième métier et dont les enfants peuvent exercer le métier du père ou d'autres métiers dont celui de cultivateur (type 4). Aux deux recensements, tant chez les ménages originaires du Québec que chez ceux originaires des 
États-Unis, le type 3 est le moins fréquent (29\%, $26 \%, 27 \%$ et $40 \%$ ), quoique ce dernier pourcentage (40 \%) indique qu'en 1881, les ménages franco-américains étaient un peu plus nombreux à combiner le métier d'agriculteur à celui de métiers rémunérés. Les ménages de type 4 sont plus nombreux aux deux recensements que ceux de type 3 , et les chefs sont plus jeunes, tant chez les Franco-américains que chez les Québécois. On peut penser que la diversification professionnelle est le fait des plus jeunes ménages. La combinaison de plusieurs métiers dans une même unité domestique serait une réponse souple de celle-ci en termes de stratégies d'adaptation. Cependant, les effectifs sont réduits et on ne peut que formuler des hypothèses.

\section{Bilan}

Alors que les Franco-américains représentent $39 \%$ (43+15/150) des unités recensées en 1891 et $32 \%(34+15 / 154)$ en 1881 et que leur fréquence chez les ménages de cultivateurs est à peu près proportionnelle à celle observée pour l'ensemble des ménages (43/101, soit $43 \%$ en 1891 et $34 / 120$, soit $28 \%$ en 1881), ils représentent seulement $31 \%(15 / 49)$ des ménages déclarant des métiers diversifiés au recensement de 1891, alors qu'ils étaient $44 \%(15 / 34)$ en 1881. Comment interpréter ces données?

D'une part, la représentation plus importante des ménages d'agriculteurs franco-américains au recensement de 1891 serait peut-être due au fait que les fils des familles franco-américains, déclarés cultivateurs chez leur père, auraient été plus nombreux que les fils de cultivateurs québécois à s'établir de façon autonome dans la même profession durant la période intercensitaire. Si oui, quels sont les facteurs qui auraient pu favoriser une plus forte stabilité de ces familles d'agriculteurs dans la communauté, ainsi qu'une persévérance plus grande dans le métier? Étant arrivés parmi les premières familles immigrantes, ces ménages auraient peut-être eu le choix d'acquérir les lots de défrichement les meilleurs et les mieux situés. D’autre part, la diminution en 1891 du nombre de ménages franco-américains déclarant exercer des professions autres qu'uniquement celle de cultivateur ( $31 \%$ contre $44 \%$ en 1881 ) pourrait être expliquée par la reprise de l'économie manufacturière aux États-Unis entre 1880 et 1890 (Lavoie, 1973) et le « retour » de certaines de ces familles, ou encore de certains de leurs membres, qui exerçaient déjà ces métiers dans les villes américaines? Le maintien des liens avec les membres de la famille restée aux États-Unis (Nadon, 1970) permettait une réponse efficace et rapide à une demande ponctuelle de main-d'œuvre et à un besoin en revenus d'appoint. Parallè- 
lement, les besoins en métiers divers d'une communauté à ce moment-là en plein développement pourraient, du moins en partie, avoir été comblés surtout par des familles québécoises, alors que certains des fils d'immigrants franco-américains demeurés dans la communauté se seraient établis plus nombreux dans le métier d'agriculteurs.

Il faut aussi noter (tableau 10) que les ménages franco-américains auraient été plus nombreux que les ménages québécois à maintenir dans l'unité domestique des fils exerçant le métier de cultivateur comme le chef de ménage, et ce, aux deux recensements ( $26 \%$ en 1891 et $38 \%$ en 1881 chez les ménages franco-américains; $19 \%$ en 1891 et $27 \%$ en 1881 chez les ménages québécois). De plus, en 1881, la différence marquée entre l'âge moyen des chefs de ménage originaires du Québec ou d'Europe (42 ans) et celui des chefs de ménage franco-américains ( 51 ans) s'expliquerait peutêtre par le fait que plusieurs des familles franco-américaines en seraient à leur deuxième migration alors que les familles venues directement du Québec ou d'Europe en seraient à leur première migration et seraient donc plus jeunes. Si les familles qui ont émigré directement des autres régions du Québec étaient plus jeunes, elles seraient aussi moins nombreuses à avoir des fils en âge de déclarer un métier et elles se classeraient dans les ménages dont la seule déclaration de métier est celle du chef. De la même manière, les familles franco-américaines, moins jeunes, devraient avoir moins de fils, voire aucun fils, se déclarant une profession, puisque ces derniers auraient déjà quitté le foyer parental. La faiblesse des effectifs nous oblige à aborder sous forme de questionnements ces premiers résultats, mais ces observations suggèrent une organisation de la famille qui serait peut-être différente selon le contexte migratoire. Il faudra, pour le confirmer, consulter des recensements antérieurs à 1881, tant canadiens qu'américains, ce qui enrichira les données produites par le jumelage des familles et des individus aux recensements de 1891 et 1881 ainsi que le jumelage avec les données de l'état civil.

\section{STRUCTURE ET COMPOSITION DES MÉNAGES}

Bien que les listes nominatives soient un pâle reflet de ce que livre l'enquête ethnologique, elles demeurent la meilleure source de données pour documenter l'organisation sociale de la communauté conjuguée au passé (Lavoie et collab., 1988, Benoist, 1989). La première étape de notre recherche, consacrée à l'analyse transversale et comparative de deux instantanés séparés par 10 années, livre des éléments intéressants à propos de l'organisation des familles et des changements qui s'y sont produits. La 
classification des unités résidentielles basée sur la typologie de Laslett (1972), typologie stimulante quoique critiquée par certains historiens (voir entre autres Berkner, 1972, 1975; Hareven, 1977a), est ici pertinente parce qu'elle s'appuie sur l'identification du ou des noyaux familiaux et de leurs extensions. Ces noyaux, même partiels, orientent la reconstitution des ascendances des fondateurs et facilitent leur jumelage aux cellules reproductives, cellules qui forment la trame généalogique fournie par le Registre de la population. Pour ces raisons, le regroupement des ménages à famille élargie avec les ménages polynucléaires en une seule catégorie de ménages complexes (ou en ménages complexes et semi-complexes) que certains historiens utilisent (Bouchard, 1994; Dessureault, 2001) n'est pas à retenir ici.

Au recensement de 1891, les instructions stipulent que l'énumérateur doit inscrire les liens qui relient les membres du ménage au chef du ménage. Outre ceux d'épouse, de fils et de fille, 20 autres liens sont spécifiés, ce qui a facilité notre classification des ménages (tableau 11). En 1881, les liens n'étant pas indiqués, la classification a été faite en tenant compte simultanément de la position dans le ménage de l'individu énuméré, de son âge et du partage ou non du même patronyme.

La grande majorité des ménages recensés sont des ménages à famille nucléaire : $83 \%$ en 1891 et $82 \%$ en 1881, la plupart avec enfants. Les ménages d'une personne et les ménages non familiaux sont les moins nombreux $(6 \%)$. Les ménages complexes, c'est-à-dire à famille élargie ou à noyau secondaire, sont un peu plus fréquents ( $11 \%$ et $12 \%)$, quoique ces deux catégories n'aient pas la même distribution aux deux recensements. Les ménages à famille étendue représentent presque le double en effectifs en 1891 de ceux à noyau secondaire, alors qu'il n'y a qu'un seul ménage polynucléaire en 1891 contre neuf en 1881 ; le noyau secondaire de ces derniers est constitué principalement d'un fils marié ou d'un gendre du chef de ménage exerçant le métier de cultivateur comme ce dernier.

Lorsque le noyau secondaire est en amont, il s'agit de parents ou de beaux-parents du chef de ménage, cultivateurs comme ce dernier. Le fait que ce type d'arrangement concerne principalement des ménages francoaméricains soulève la possibilité qu'il soit l'héritage d'une organisation de la famille adaptée à la vie de locataires dans des logements d'entreprise ou à proximité des quartiers francophones des villes industrielles de la Nouvelle-Angleterre (Hareven, 1977b). Cette organisation peut également témoigner de la décision de revenir au Québec à plusieurs familles apparentées afin de partager la vie de défricheurs en faisant appel au système d'entraide basé sur la parenté (Collard, 1999), système qui caractérise aussi le comportement migratoire des émigrants québécois dans les États industriels de l'Est américain (Hareven, 1977b ; Roby, 2000). 
TABLEAU 11 Structure des ménages recensés en 1891 et en 1881 selon la typologie de Laslett

\begin{tabular}{|c|c|c|}
\hline \multirow[t]{2}{*}{ Types de ménage } & \multicolumn{2}{|c|}{ Nombre de ménages } \\
\hline & 1891 & 1881 \\
\hline Ménage d'une personne & 8 & 6 \\
\hline Ménage sans structure familiale & 1 & 3 \\
\hline \multicolumn{3}{|l|}{ Ménage simple ${ }^{a}$} \\
\hline Couple sans enfant & 15 & 9 \\
\hline Couple avec enfant & 103 & 109 \\
\hline Femme seule avec enfant & 6 & 2 \\
\hline Homme seul avec enfant & 2 & 7 \\
\hline \multicolumn{3}{|l|}{ Ménage à famille élargie } \\
\hline Avec extension en amont & 9 & 5 \\
\hline Avec extension en amont et latérale & 1 & o \\
\hline Avec extension en aval & 3 & o \\
\hline Avec extension en aval et en amont & 1 & o \\
\hline Avec extension latérale & 2 & 4 \\
\hline \multicolumn{3}{|l|}{ Ménage polynucléaire } \\
\hline avec unité secondaire en aval & 1 & 7 \\
\hline avec unité secondaire en amont & o & 2 \\
\hline TOTAL & 152 & 154 \\
\hline
\end{tabular}

a 1891: dont 5 ménages avec étranger(s) a 1881: dont 3 ménages avec étranger(s)

Quant aux extensions du noyau familial, les extensions en amont sont les plus nombreuses en 1891 et il s'agit principalement de ménages accueillant un parent ou un des beaux-parents du chef de ménage. Les extensions latérales sont rares en 1891 et elles caractérisent deux ménages avec peu d'enfants à la maison, et au sein desquels vit un beau-frère menuisier et un autre employé chez le chef déclaré cultivateur-hôtelier. En 1881, les extensions latérales sont dues à la présence d'un jeune frère d'un chef cultivateur dont les enfants sont trop jeunes pour être en âge de travailler. Les membres qui composent l'extension sont déclarés cultivateurs, à l'exception d'un journalier. Dans tous les cas, ce type d'extension relève de l'ajout d'un membre adulte qui travaille et participe à l'économie du ménage. Ce type d'aide ne surprend pas dans un contexte de peuplement, où toute l'aide que la parenté peut fournir sera encouragée. L'extension en aval n'est représentée qu'en 1891 et elle est due à la présence d'un petit-fils et de neveux, dont un est commis chez son oncle marchand général. Toutes 
ces extensions se retrouvent chez les ménages tant franco-américains que québécois.

\section{CONCLUSION}

Les recensements de 1881 et de 1891 ont été choisis pour amorcer notre étude des fondateurs de la population de La Patrie parce qu'ils suivent de près la fondation officielle de la colonie à la suite de la promulgation de l'Acte de rapatriement en 1875 . Ils offrent également des données sur l'arrivée des premiers colons recrutés quelque temps auparavant par les sociétés de colonisation. Il est légitime de considérer que les familles recensées sont bien celles des premiers fondateurs de la colonie.

L'analyse de la structure par âge et par sexe des deux populations confirme qu'elles partagent un profil commun de population jeune, qui correspond à ce que l'on peut attendre d'une population récemment fondée par des familles immigrantes en âge de s'établir sur des terres neuves. L'augmentation en 1891 du nombre d'individus de 60 ans et plus confirmerait l'établissement de certaines familles et la fin de la période de colonisation comme telle. Les familles immigrantes auraient été accompagnées d'un plus grand nombre de fils que de filles, ce dont témoigne le rapport de masculinité pour le groupe d'âge des 10-39 en 1881, rapport qui se serait maintenu en 1891 et qui peut s'expliquer par les cinq scieries pleinement fonctionnelles en 1890. Ces dernières auraient assuré la présence d'une main d'œuvre principalement masculine, qu'elle provienne des familles déjà sur place, du retour des États-Unis de certains de ses membres ou bien de nouvelles familles attirées par le développement économique du canton.

La déclaration du lieu de naissance de chaque individu confirme l'arrêt de l'immigration européenne dans le canton et le départ des familles originaires d'Europe, ainsi que l'arrivée récente de familles en provenance des États américains et de paroisses et de villes du Québec. C’est cependant l'analyse des ménages comme unité d'observation qui documente le mieux la contribution franco-américaine et québécoise à la population de La Patrie. Cette analyse selon différentes combinaisons des lieux de naissance des membres des ménages conduit à une estimation minimale de $39 \%$ (1891) et $32 \%$ (1881) de la fréquence des ménages franco-américains, ce qui illustre l'importance des allers-retours transfrontaliers des familles. L'évaluation du nombre de familles franco-américaines basée sur une définition souple exclut les ménages dont tous les membres sont nés au Québec, ce qui fait que les ménages franco-américains sont probablement sous- 
estimés, car un séjour de la famille aux États-Unis n'a pas été nécessairement marqué par la naissance d'un enfant en sol américain. L'examen des écarts d'âge entre les membres du ménage et des intervalles entre les naissances peut suggérer des séjours aux États-Unis, sans que ce soit toutefois la seule explication possible.

Même si la variété des professions est plus grande en 1891, il n'en reste pas moins que la population se définit principalement par les activités agricoles aux deux recensements, et ce, en dépit de l'importance de l'industrie forestière dans la vie et la survie de la communauté. Considéré principalement comme fournissant un revenu d'appoint, le travail de forestier n'aurait ainsi pas fait l'objet de déclaration de profession, à quelques exceptions près en 1891. L'examen de la distribution des professions au sein des ménages suggère une organisation du travail qui serait quelque peu différente selon l'origine des familles, quoique les petits effectifs imposent la prudence dans l'interprétation. Les ménages de cultivateurs francoaméricains seraient plus nombreux que les ménages québécois à garder dans l'unité domestique des fils exerçant le même métier que celui de leur père, et ce, aux deux recensements, mais de façon plus marquée en 1881. Ces familles franco-américaines auraient aussi réussi à établir plus de fils cultivateurs en 1891 que les familles québécoises. Par ailleurs, si l'on considère l'ensemble des types d'arrangements professionnels autres que celui des cultivateurs à part entière, ce sont les ménages québécois, en 1891, qui seraient les plus nombreux à recourir à cette forme d'organisation du travail au sein de la famille.

Quant aux unités recensées, elles sont principalement composées d'un noyau familial avec enfants, bien que la présence de ménages à famille élargie ou à plus d'un noyau révèle une organisation familiale un peu plus complexe, et différente aux deux recensements. La présence quasi exclusive, lors de l'énumération de 1881, de ménages à noyau secondaire formé d'une jeune famille, de même que le nombre plus important de maisons habitées par plusieurs ménages, caractérisent la période d'établissement dans la colonie. Cette cohabitation de plusieurs ménages, révélée par le numéro séquentiel attribué par le recenseur à chaque habitation (non relevé au dépouillement fait par les mormons), ainsi que le partage fréquent d'un même patronyme suggèrent des arrangements basés sur les liens de parenté. Le fait que les chefs de ménage des habitations voisines portent souvent le même patronyme appuie cette hypothèse d'un type d'organisation en voisinage basée sur les liens familiaux. La prédominance, en 1891, de ménages accueillant un ou les deux parents âgés du chef de ménage ou de son épouse est cohérente avec la présence dans la population 
d'un plus grand nombre de personnes âgées. La plus grande variété des types de ménage à famille étendue témoigne d'arrangements à interpréter dans un contexte d'allers-retours migratoires.

Le point le plus important à débattre n'est pas tant la question de l'extension de ces résultats à l'ensemble de la région quant à la contribution des rapatriés franco-américains à la population des Cantons-de-l'Est que la compréhension des dynamiques familiales et des stratégiques utilisées par les familles immigrantes pour assurer leur survie et leur enracinement ou non dans la région. C'est ce qui contribuera à améliorer notre compréhension de la colonisation de cette région du Québec et de la formation des pools géniques régionaux. Par ailleurs, un des intérêts méthodologiques de l'ensemble du programme de recherche est la possibilité de comparer les résultats de l'analyse transversale à ceux obtenus par analyse longitudinale et par conjugaison des approches transversales et longitudinales. 
ANNEXE A Nombre de ménages susceptibles d'avoir fait des séjours aux États-Unis

\begin{tabular}{|l|c|c|c|c|}
\hline Groupes d'âge & \multicolumn{2}{|c|}{$\begin{array}{c}\text { Nombre de ménages dont tous } \\
\text { les enfants sont nés au Québec }\end{array}$} & \multicolumn{2}{|c|}{$\begin{array}{c}\text { Nombre de ménages susceptibles } \\
\text { d'avoir fait des séjours aux États-Unis }\end{array}$} \\
\hline & $\mathbf{1 8 9 1}$ & $\mathbf{1 8 8 1}$ & $\mathbf{1 8 9 1}$ & $\mathbf{1 8 8 1}$ \\
\hline 20-29 ans & 12 & 21 & 2 & 1 \\
\hline 30-39 ans & 26 & 20 & 2 & 1 \\
\hline 40-49 ans & 13 & 16 & 3 & 3 \\
\hline 50-59 ans & 13 & 14 & 6 & 8 \\
\hline 60 ans et plus & 5 & 4 & 2 & 4 \\
\hline TOTAL & $\mathbf{6 9}$ & $\mathbf{7 5}$ & $\mathbf{1 5}$ & $\mathbf{1 7}$ \\
\hline
\end{tabular}

\begin{tabular}{|l|c|c|c|c|}
\hline Groupes d'âge & \multicolumn{2}{|c|}{$\begin{array}{c}\text { Nombre de ménages dont } \\
\text { tous les enfants sont nés } \\
\text { aux États-Unis }\end{array}$} & \multicolumn{2}{c|}{$\begin{array}{c}\text { Nombre de ménages susceptibles } \\
\text { d'avoir fait des séjours aux États-Unis }\end{array}$} \\
\hline & $\mathbf{1 8 9 1}$ & $\mathbf{1 8 8 1}$ & $\mathbf{1 8 9 1}$ & $\mathbf{1 8 8 1}$ \\
\hline $20-29$ ans & 1 & 0 & 0 & 0 \\
\hline 30-39 ans & 1 & 0 & 0 & 0 \\
\hline 40-49 ans & 1 & 0 & 0 & 0 \\
\hline 50-59 ans & 2 & 1 & 1 & 1 \\
\hline 60 ans et plus & 2 & 0 & 2 & 0 \\
\hline TOTAL & $\mathbf{7}$ & $\mathbf{1}$ & $\mathbf{3}$ & $\mathbf{1}$ \\
\hline
\end{tabular}

\begin{tabular}{|l|c|c|c|c|}
\hline Groupes d'âge & \multicolumn{2}{|c|}{$\begin{array}{c}\text { Nombre de ménages dont } \\
\text { les enfants sont nés } \\
\text { principalement en Europe }\end{array}$} & \multicolumn{2}{c|}{$\begin{array}{c}\text { Nombre de ménages susceptibles } \\
\text { d'avoir fait des séjours aux États-Unis }\end{array}$} \\
\hline & $\mathbf{1 8 9 1}$ & $\mathbf{1 8 8 1}$ & $\mathbf{1 8 9 1}$ & $\mathbf{1 8 8 1}$ \\
\hline 20-29 ans & 0 & 0 & 0 & 0 \\
\hline $30-39$ ans & 1 & 2 & 0 & 0 \\
\hline $40-49$ ans & 0 & 5 & 0 & 0 \\
\hline 50-59 ans & 0 & 3 & 0 & 1 \\
\hline 60 ans et plus & 2 & 1 & 2 & 1 \\
\hline TOTAL & $\mathbf{3}$ & $\mathbf{1 1}$ & $\mathbf{2}$ & $\mathbf{2}$ \\
\hline
\end{tabular}


ANNEXE A (suite)

\begin{tabular}{|l|c|c|c|c|}
\hline Groupes d'âge & \multicolumn{2}{|c|}{$\begin{array}{c}\text { Nombre de ménages avec } \\
\text { diverses combinaisons } \\
\text { d'aller-retour Québec É.-U. }\end{array}$} & \multicolumn{2}{|c|}{$\begin{array}{c}\text { Nombre de ménages susceptibles } \\
\text { d'avoir fait plus de séjours aux États- } \\
\text { Unis }\end{array}$} \\
\hline & $\mathbf{1 8 9 1}$ & $\mathbf{1 8 8 1}$ & $\mathbf{1 8 9 1}$ & $\mathbf{1 8 8 1}$ \\
\hline 20-29 ans & 6 & 6 & 0 & 0 \\
\hline 30-39 ans & 16 & 19 & 0 & 5 \\
\hline 40-49 ans & 9 & 10 & 0 & 3 \\
\hline 50-59 ans & 5 & 10 & 0 & 9 \\
\hline 60 ans et plus & 8 & 2 & 5 & $\mathbf{2}$ \\
\hline TOTAL & $\mathbf{4 4}$ & $\mathbf{4 7}$ & $\mathbf{5}$ & $\mathbf{1 9}$ \\
\hline
\end{tabular}

\begin{tabular}{|l|c|c|c|c|}
\hline Groupes d'âge & \multicolumn{2}{|c|}{$\begin{array}{c}\text { Nombre de ménages } \\
\text { sans enfant }\end{array}$} & \multicolumn{2}{c|}{$\begin{array}{c}\text { Nombre de ménages susceptibles } \\
\text { d'avoir fait des séjours aux États-Unis }\end{array}$} \\
\hline & $\mathbf{1 8 9 1}$ & $\mathbf{1 8 8 1}$ & $\mathbf{1 8 9 1}$ & $\mathbf{1 8 8 1}$ \\
\hline 20-29 ans & $13^{\text {a }}$ & $9^{\text {b }}$ & 0 & 0 \\
\hline 30-39 ans & 3 & 4 & 0 & 0 \\
\hline 40-49 ans & 3 & 2 & 0 & 1 \\
\hline 50-59 ans & 2 & 3 & 1 & 1 \\
\hline 60 ans et plus & 8 & 2 & 6 & 2 \\
\hline TOTAL & $\mathbf{2 9}$ & $\mathbf{2 0}$ & $\mathbf{7}$ & $\mathbf{4}$ \\
\hline
\end{tabular}

a dont 5 célibataires, 7 couples et un veuf

b dont un couple dont les deux conjoints ont 18 ans; 2 célibataires de 18 et 19 ans 
ANNEXE B Professions déclarées aux recensements de 1891 et 1881

\begin{tabular}{|c|c|c|c|c|c|c|c|c|}
\hline \multirow[b]{2}{*}{ PROFESSION } & \multicolumn{4}{|c|}{ Recensement de 1891} & \multicolumn{4}{|c|}{ Recensement de 1881} \\
\hline & CHEF & $\begin{array}{l}\text { FILS/ } \\
\text { FILLE }\end{array}$ & $\begin{array}{c}\text { AUTRE/ } \\
\text { SANS } \\
\text { LIEN }\end{array}$ & TOTAL & CHEF & FILS & AUTRE & TOTAL \\
\hline Boucher & 1 & 1 & & 2 & & & & \\
\hline Charron & 1 & & & 1 & 1 & & & 1 \\
\hline Commis & & & 1 & 1 & & & & \\
\hline Apprenti cordonnier & & & & & & 1 & & 1 \\
\hline Cordonnier & 1 & 1 & & 2 & & & & \\
\hline Cultivateur fermier & 1 & & & 1 & & & & \\
\hline Cultivateur forgeron & & & & & 2 & & & 2 \\
\hline Cultivateur hôtelier & 1 & & & 1 & & & & \\
\hline Cultivateur menuisier & 1 & & & 1 & 1 & & & 1 \\
\hline Cultivateur peintre & & & & & 1 & & & 1 \\
\hline Cultivateur & 115 & 36 & $4^{e}$ & 155 & 124 & 21 & 4 & 149 \\
\hline Fils de cultivateur & & & & & 2 & 58 & & 60 \\
\hline Domestique & & & $1^{d}$ & 1 & & & & \\
\hline Employé & & & 1 & 1 & & & & \\
\hline Étudiant & & 2 & & 2 & & & & \\
\hline Fermier & 2 & & & 2 & 4 & 2 & & 6 \\
\hline Fils de fermier & & & & & & 1 & & 1 \\
\hline Fileuse & & 5 & & 5 & & & & \\
\hline Forestier & 4 & 5 & & 9 & & 1 & & 1 \\
\hline Forgeron & 2 & 1 & & 3 & 1 & 1 & & 2 \\
\hline Imprimeur & & & & & & & 1 & 1 \\
\hline Institutrice & 2 & 1 & $1+2^{d}$ & 6 & 2 & & $2^{c}$ & 4 \\
\hline Journalier & & 1 & & 1 & 2 & 7 & 2 & 11 \\
\hline Journalier de ferme & & 1 & & 1 & & & 1 & 1 \\
\hline Maçon & 1 & & & 1 & & & & \\
\hline Maison de pension & 1 & & & 1 & & & & \\
\hline Marbrier & 1 & & & 1 & & & & \\
\hline Marchand & 3 & 1 & & 4 & 1 & & & 1 \\
\hline Marchand cordonnier & & & & & 1 & & & 1 \\
\hline Marchand général & 1 & & & 1 & & & & \\
\hline Marinier & & & & & & & 1 & 1 \\
\hline Médecin chirurgien & 1 & & & 1 & & & & \\
\hline
\end{tabular}


ANNEXE B Professions déclarées aux recensements de 1891 et 1881 (suite)

\begin{tabular}{|c|c|c|c|c|c|c|c|c|}
\hline \multirow[b]{2}{*}{ PROFESSION } & \multicolumn{4}{|c|}{ Recensement de 1891} & \multicolumn{4}{|c|}{ Recensement de 1881} \\
\hline & CHEF & $\begin{array}{l}\text { FILS/ } \\
\text { FILLE }\end{array}$ & $\begin{array}{c}\text { AUTRE/ } \\
\text { SANS } \\
\text { LIEN }\end{array}$ & TOTAL & $\mathrm{CHEF}$ & FILS & AUTRE & TOTAL \\
\hline Mécanicien & 3 & & & 3 & & & & \\
\hline Ménagère & & & $1^{\mathrm{d}}$ & 1 & & & 1 & 1 \\
\hline Menuisier & & 4 & 1 & 5 & 2 & & & 2 \\
\hline Menuisier contracteur & & & & & 1 & & & 1 \\
\hline Mineur & 2 & & & 2 & & 1 & & 1 \\
\hline Moine trappiste & & & & & 1 & & & 1 \\
\hline Ouvrier de ferme & 1 & 2 & & 3 & & & 1 & 1 \\
\hline Ouvrière de ferme & 1 & & & 1 & & & & \\
\hline Prop. de moulin & 1 & & & 1 & 2 & & & 2 \\
\hline Prêtre curé & 1 & & & 1 & 1 & & & 1 \\
\hline Rentier & 1 & & $2+1^{4}$ & 4 & 2 & & & 2 \\
\hline Rentière & 1 & 1 & & 2 & & & & \\
\hline Secrétaire municipal & & & & & 1 & & & 1 \\
\hline Sellier & & 1 & & 1 & & & & \\
\hline Servante & & & $1^{4}$ & 1 & & & 1 & 1 \\
\hline Tanneur & & & & & & 1 & & 1 \\
\hline Sans profession & $2^{\mathrm{a}}$ & & & & $2^{b}$ & & & \\
\hline TOTAL & 150 & 63 & 16 & 229 & 152 & 94 & 14 & 260 \\
\hline
\end{tabular}

${ }^{a}$ Deux chefs de ménage âgés de 68 ans et 24 ans déclarés «patron» dans la rubrique 17 du recensement;

b Deux veuves dont les fils sont cultivateurs et fils de cultivateurs;

c Une épouse et une fille;

d Sans lien;

e Dont une épouse.

En grisé: les professions absentes au recensement de1891/absentes au recensement de 1881 


\section{BIBLIOGRAPHIE}

Anctil, P. 1980. Aspect of Class Ideology in the New England Ethnic Minority: the Franco-Americans of Woonsocket, Rhode Island (1865-1929). Thèse de doctorat, New-York University, New School for Social Research.

Annuaire du séminaire St Charles Borromée, Sherbrooke, 1897-1898, vol. IV, $\mathrm{n}^{\circ} 3,23$, Imprimerie du Séminaire. Chap. XI, Notes historiques sur les paroisses $d u$ diocèse de Sherbrooke. La colonie de rapatriement, partie I : 237-291.

Annuaire du séminaire St Charles Borromée, Sherbrooke, 1898-1899, vol. IV, $\mathrm{n}^{\circ}$ 4, 24, Imprimerie du Séminaire, Chap. XI, Notes historiques sur les paroisses $d u$ diocèse de Sherbrooke. La colonie de rapatriement, partie II : 391-408.

Annuaire du séminaire St Charles Borromée, Sherbrooke. 1896-9. Notes historiques sur les paroisses du diocèse de Sherbrooke, cité dans J.I. LitTLE (1977), note 86, et reproduit pour les pages 279-280 par Y. VézInA, Comité du centenaire de La Patrie, 1975: 13-14.

Benoist, J. 1989. "Saint Barthélemy : racines et destins d'une population » dans Singaravélou (dir.), Pauvreté et développement dans les pays tropicaux, Bordeaux, CEGET (Centre d'Études de Géographie Tropicale-CNRD), Université de Bordeaux III : 305-317.

Berkner, L. K. 1972. "The stem family and the developmental cycle of the peasant household : an eighteenth-centurey Austrian example », American Historical Review, 77, 1-2:398-418.

BERKNER, L. K. 1975. "The use and misuse of census data in the historical study of family structure ", Journal of Interdisciplinary History, $5:$ 721-763.

Bibliothèque et Archives CANAdA, Le recensement du Canada (1881)/Aide à la recherche/base de données. http ://www.collectionscanada.gc.ca/base-dedonnees/recensement-1881/o01049-300o-f.html

Bouchard, G. 1977. «Introduction à l'étude de la société saguenayenne aux XIX ${ }^{\mathrm{e}}$ et $\mathrm{xx}^{\mathrm{e}}$ siècles ", Revue d'histoire de l'Amérique française, 31, 1:3-27.

Bouchard, G. 1978. "Démographie et société rurale au Saguenay, 1851-1935 ", Recherches sociographiques, 19, $1:$ 7-31.

Bouchard, G. 1987. "Sur la reproduction familiale en milieu rural : systèmes ouverts et systèmes clos", Recherches sociographiques, 28, 2-3: 229-251.

Bouchard, G. 1992. «Les migrations de réallocation comme stratégie de reproduction familiale en territoire neuf» dans R. Bonnain, J. Goy et G. Bouchard (dir.), Transmettre, hériter, succéder. La reproduction familiale en milieu rural: France-Québec, XVIII ${ }^{e}-X^{e}$ siècles, Lyon et Paris, Presses universitaires de Lyon et École des hautes études en sciences sociales : 189-212.

Bouchard, G. 1994. «Pressions agraires et structures des ménages au Saguenay (1881-1931)», European Journal of Population, 10 : 175-197.

CARtagène, http ://www.cyberpresse.ca/actualites/quebec-canada/sante/200908/. P. Breton, Une carte génétique du Québec. 
Charbonneau, H. 1991. "Les francophones du Québec de 1608 à 1960 », dans J. Henripin et Y. Martin (dir.), La population du Québec d'hier à demain, Montréal, Les Presses de l'Université de Montréal.

Collard, C. 1999. Une famille, un village, une nation. La parenté dans Charlevoix, 1900-1960. Cap-Saint-Ignace, Les éditions du Boréal, 194 p.

Comité du centenaire de La Patrie. 1975. Centenaire de La Patrie, 1875-1975. Sherbrooke, Éditions Pauline, 173 pages.

DAhlberg, G. 1929. "Inbreeding in man », Genetics, 14: 421-454.

Département de l'agriculture (Bureau du recensement). 1881. Manuel contenant "l'acte du recensement " et les instructions aux officiers du deuxième recensement du Canada (1881). Ottawa, Brown Chamberlin, 53 pages.

Département de l'agriculture (Division du recensement). 1891. Manuel contenant "l'acte du recensement » et les instructions aux officiers employés à faire le troisième recensement du Canada (1891). Ottawa, Brown Chamberlin, 35 pages.

Dessureault, C. 2001. "Parenté et stratification sociale dans une paroisse rurale de la vallée du Saint-Laurent au milieu du XIx ${ }^{\mathrm{e}}$ siècle ", Revue d'histoire de l'Amérique française, 54, $3:$ 411-447.

Dobzhansky, T. 1950. Genetics of the Evolutionary Process. New York, Columbia University Press, 505 pages.

Gomila, J. et L. Guyon. 1969. "Étude comparative de petites communautés rurales; méthode et premiers résultats à propos de la consanguinité à Bois-Vert (Québec) », Population, 24, 6:1127-1153.

Gomila, J. 1971. "Définir la population », dans J. Jacquard (dir.), L'étude des isolats, espoirs et limites, Paris, Association anthropologique internationale de langue française, École pratique des Hautes Études et Institut national d'études démographiques : 5-36.

Hareven, T. K. 1977a. "The family as process : the historical study of family cycle », Journal of Family History, $7:$ 322-329.

Hareven, T.K. 1977b. "Family time and industrial time : family and work in a planned corporation town, 1900-1924» dans K. Hareven (dir.), Family and kin in urban communities, 1700-1930, New York, Franklin Watts.

Heyer, É. 1999. "One founder/one gene hypothesis in a new expanding population : Saguenay (Québec, Canada) », Human Biology, 71, 1 : 99-109.

JACQUARD, A. et R. NADOT. 1968. " Mariages consanguins et fertilité différentielle », Population, 23, $2:$ 325-332.

JaCQUARD, A. 1972. «Évolution du patrimoine génétique des Kel Kummer ». Population, 4, 5:784-800.

Jacquard, A. 1977. Concepts en génétique des populations, Paris, Masson, Collection de biologie évolutive 4 . 
Lavoie, Y. 1973. "Les mouvements migratoires des Canadiens entre leur pays et les États-Unis au XIX ${ }^{\mathrm{e}}$ et au $\mathrm{xx}^{\mathrm{e}}$ siècles : étude quantitative », dans H. CHARBONNEAU (dir.), La population du Québec : études rétrospectives : 73-88.

Lavoie, Y., J. Benoist et F. M. Mayer. 1988. «Du synchronique au diachronique: méthodes d'étude cumulative de recensements nominatifs ", Bulletins et Mémoires de la Société d'anthropologie de Paris, $\mathrm{XIV}^{\mathrm{e}}$ série, tome 5, fascicule 1-2: 61-70.

LASLETT, P. 1972. «Introduction : the History of the Family », dans P. Laslett (dir.), Household and Family in Past Time, Cambridge, Cambridge University Press : 1-89.

Linteau, P. A. 2000. "Les migrants américains et franco-américains au Québec, 1792-1940: un état de la question ", Revue d'histoire de l'Amérique française, 53, $4: 561-602$.

Little, J. I. 1977. "La Patrie: Québec's Repatriation Colony, 1875-1880 », dans Canadian Historical Association/Société historique du Canada, Historical Papers/Communications historiques : 67-85.

Maisonneuve, D. 1985. "Structure familiale et exode rural; le cas de SaintDamase, 1852-1861 ", Cahiers québécois de démographie, 14, 2 : 231-240.

Mathieu, J., P. Therrien-Fortier et R. Lessard. 1987. " Mobilité et sédentarité; stratégies familiales en Nouvelle-France »,. Recherches sociographiques, 28, 2-3: 211-227.

MAYER, F. M. 1977. Évolution de la structure génétique d'une population ouverte : La Serpentine. Thèse de doctorat, Département d'anthropologie, Faculté des arts et des sciences, Université de Montréal, 685 pages.

Nadon, P. 1970. Étude des principaux mécanismes d'intégration d'une communauté canadienne-française. Mémoire de maîtrise, Département d'anthropologie, Faculté des sciences sociales, Université de Montréal, Montréal, 301 pages.

Ramirez, B. 1991. Par monts et par vaux. Migrants canadiens-français et italiens dans l'économie nord atlantique, 1860-1914. Montréal, Éditions du Boréal, 204 pages.

Ramirez, B. et J. Lamarre, 1985. «Du Québec vers les États-Unis : l'étude des lieux d'origine " [note de recherche], Revue d'histoire de l'Amérique française, $38,3: 409-422$.

Rову, Y. 2000. Les franco-américains de la Nouvelle-Angleterre. Rêves et réalités. Sillery, Québec, Les Éditions du Septentrion, 526 pages.

Rodrigue, M. 1997. Les franco-américains à Montréal au début du XXe siècle. Mémoire de maîtrise en histoire, Université du Québec à Montréal, Montréal, 120 pages.

SÉGuin, N., 1977. La conquête du sol au XIXe siècle, Sillery, Boréal Express, 296 pages. St-Hilaire, M. 1996. Peuplement et dynamique migratoire au Saguenay, 1840-1960. Sainte-Foy, Les Presses de l'Université Laval, 285 p. 
Thompson, L. 1967. "Steps toward a unified anthropology», Current Anthropo$\log y, 8,1-2: 67-91$.

Vézina, Y. 1975. "La colonie de rapatriement », dans Comité du Centenaire de La Patrie, Centenaire de La Patrie, 1875-1975, Sherbrooke, Éditions Pauline : 13-15.

Wapples, R. S. et O. Caggiotti. 2006. "What is a population? An empirical evaluation of some genetic methods for identifying the number of gene pools and their degree of connectivity ", Molecular Ecology, 15 : 1419-1439.

\section{ABSTRACT}

\section{Francine M. MAYER and Mireille BOISVERT}

The biological founders of the population of La Patrie in the Eastern Townships: FrancoAmericans, Québécois and Europeans in the 1881 and 1891 Canadian Censuses

The research results presented here are the first of a large interdisciplinary research program on the genetic contribution of the Franco-American immigrants to the gene pool of the population of the Eastern Townships and more specifically to the gene pool of the population of La Patrie, founded in 1875 following the adoption by the Québec government of the Act of Repatriation. This act promoted the return of French-Canadians who had emigrated to the industrial towns of New England to work primarily in the textile mills. In order to understand the complex interaction of the biological and social factors that contributed to shaping the population gene pools we began the research with transversal and comparative analyses of the 1881 and 1891 Canadian censuses. Since these were taken immediately after the arrival of the first settlers, their analysis allows us to identify the first biological founders of the population and to arrive at a first estimate of the number of Franco-American families which represent, at each census, more than one third of the enumerated households. Demographic structures indicate a young population with a high sex ratio for the 10-39 age group suggesting that the families arrived with more sons than daughters at working age, in order to help clear their allocated lands for cultivation. Alternations in the declaration of birthplace for each household member reveal an important mobility across the national borders for a number of families. Professions are more diversified in 1891 but agriculture is still dominant while employment in the lumbering industries remained a security valve for the settlers. 